\title{
Twisted Modules for Toroidal Vertex Algebras
}

\author{
Fei Kong ${ }^{a}$, Haisheng Li国, Shaobin Tan² and Qing Wang $₫ 3$ \\ ${ }^{a}$ School of Mathematical Sciences, Xiamen University, Xiamen 361005, China \\ ${ }^{b}$ Department of Mathematical Sciences, \\ Rutgers University, Camden, NJ 08102, USA
}

\begin{abstract}
This is a paper in a series systematically to study toroidal vertex algebras. Previously, a theory of toroidal vertex algebras and modules was developed and toroidal vertex algebras were explicitly associated to toroidal Lie algebras. In this paper, we study twisted modules for toroidal vertex algebras. More specifically, we introduce a notion of twisted module for a general toroidal vertex algebra with a finite order automorphism and we give a general construction of toroidal vertex algebras and twisted modules. We then use this construction to establish a natural association of toroidal vertex algebras and twisted modules to twisted toroidal Lie algebras. This together with some other known results implies that almost all extended affine Lie algebras can be associated to toroidal vertex algebras.
\end{abstract}

\section{Introduction}

Extended affine Lie algebras are natural generalizations of affine Kac-Moody Lie algebras (see AABGP ), where an important family of extended affine Lie algebras consists of toroidal Lie algebras which are central extensions of multi-loop algebras of finite dimensional simple Lie algebras. It is known that (untwisted) affine Lie algebras can be canonically associated with vertex algebras and modules (cf. [FZ], [DL], [Li1], [LL]), while twisted affine Lie algebras can be associated with vertex algebras in terms of twisted modules (see [FLM], Li2]). In [BBS, a natural connection of certain toroidal Lie algebras with vertex algebras was established. On the other hand, it is natural to study suitable toroidal analogues of vertex algebras and their relations with toroidal Lie algebras from a different perspective. This is also potentially important from the viewpoint of physics conformal field theory in high dimensions (cf. [IKU], [IKUX]). With this as the main driving force, a theory of toroidal vertex algebras and modules was developed and toroidal vertex algebras and their modules were associated to toroidal Lie algebras in [LTW2].

In the current paper, we continue to study twisted modules for toroidal vertex algebras, with a goal to associate twisted modules for certain toroidal vertex algebras to modules for twisted toroidal Lie algebras. To achieve this goal, we develop

\footnotetext{
${ }^{1}$ Partially supported by China NSF grant (No. 11471268)

${ }^{2}$ Partially supported by China NSF grant (No. 11471268)

${ }^{3}$ Partially supported by China NSF grant (No. 11371024), Natural Science Foundation of Fujian Province (No. 2013J01018) and Fundamental Research Funds for the Central University (No. 2013121001).
} 
a theory of twisted modules for a toroidal vertex algebra with a finite order automorphism and we establish a conceptual construction of toroidal vertex algebras and twisted modules. By using this general result, we successfully associate twisted modules for certain toroidal vertex algebras to twisted toroidal Lie algebras.

Note that affine Kac-Moody algebras were classified as untwisted affine Lie algebras and twisted affine Lie algebras, where untwisted affine Lie algebras can be realized as the universal central extensions of loop algebras of finite dimensional simple Lie algebras and twisted affine Lie algebras can be realized as fixed points subalgebras of untwisted affine Lie algebras under Dynkin digram automorphisms (see $[\mathrm{K}]$ ). For extended affine Lie algebras, essentially this is also the case; it was proved (see [ABFP, N1, N2, BGK, BGKN, Y] ) that almost all extended affine Lie algebras (except those constructed from the centerless irrational Lie tori) can be realized as twisted toroidal Lie algebras. In view of this, almost all extended affine Lie algebras can be associated to toroidal vertex algebras.

Now, we give a more detailed account of the contents of this paper. First, an $(r+1)$-toroidal vertex algebra (with $r$ a positive integer) is defined (see [LTW2]) to be a vector space $V$ equipped with a linear map

$$
\begin{aligned}
Y\left(\cdot ; x_{0}, \mathbf{x}\right): \quad & V \rightarrow \operatorname{Hom}\left(V, V\left[\left[x_{1}^{ \pm 1}, \ldots, x_{r}^{ \pm 1}\right]\right]\left(\left(x_{0}\right)\right)\right) \\
& v \mapsto Y\left(v ; x_{0}, \mathbf{x}\right),
\end{aligned}
$$

where $\mathbf{x}=\left(x_{1}, \ldots, x_{r}\right)$, and equipped with a distinguished vector $\mathbf{1}$ of $V$, such that

$$
Y\left(\mathbf{1} ; x_{0}, \mathbf{x}\right) v=v \text { and } Y\left(v ; x_{0}, \mathbf{x}\right) \mathbf{1} \in V\left[\left[x_{0}, x_{1}^{ \pm 1}, \ldots, x_{r}^{ \pm 1}\right]\right] \quad \text { for } v \in V,
$$

and such that for $u, v \in V$,

$$
\begin{gathered}
z_{0}^{-1} \delta\left(\frac{x_{0}-y_{0}}{z_{0}}\right) Y\left(u ; x_{0}, \mathbf{z y}\right) Y\left(v ; y_{0}, \mathbf{y}\right)-z_{0}^{-1} \delta\left(\frac{y_{0}-x_{0}}{-z_{0}}\right) Y\left(v ; y_{0}, \mathbf{y}\right) Y\left(u ; x_{0}, \mathbf{z y}\right) \\
=y_{0}^{-1} \delta\left(\frac{x_{0}-z_{0}}{y_{0}}\right) Y\left(Y\left(u ; z_{0}, \mathbf{z}\right) v ; y_{0}, \mathbf{y}\right) .
\end{gathered}
$$

Now, let $V$ be an $(r+1)$-toroidal vertex algebra with a finite order automorphism $\sigma$ of period $N$. We define a $\sigma$-twisted $V$-module to be a vector space $W$ equipped with a linear map

$$
\begin{aligned}
Y_{W}\left(\cdot ; x_{0}, \mathbf{x}\right): \quad & V \rightarrow \operatorname{Hom}\left(W, W\left[\left[x_{1}^{ \pm 1}, \ldots, x_{r}^{ \pm 1}\right]\right]\left(\left(x_{0}^{\frac{1}{N}}\right)\right)\right) \\
& v \mapsto Y_{W}\left(v ; x_{0}, \mathbf{x}\right)
\end{aligned}
$$

such that

$$
Y_{W}\left(\mathbf{1} ; x_{0}, \mathbf{x}\right)=1_{W}
$$

and for $u, v \in V$,

$$
\begin{gathered}
z_{0}^{-1} \delta\left(\frac{x_{0}-y_{0}}{z_{0}}\right) Y_{W}\left(u ; x_{0}, \mathbf{z y}\right) Y_{W}\left(v ; y_{0}, \mathbf{y}\right)-z_{0}^{-1} \delta\left(\frac{y_{0}-x_{0}}{-z_{0}}\right) Y_{W}\left(v ; y_{0}, \mathbf{y}\right) Y_{W}\left(u ; x_{0}, \mathbf{z y}\right) \\
=\frac{1}{N} \sum_{j=0}^{N-1} y_{0}^{-1} \delta\left(\omega_{N}^{j}\left(\frac{x_{0}-z_{0}}{y_{0}}\right)^{\frac{1}{N}}\right) Y_{W}\left(Y\left(\sigma^{j} u ; z_{0}, \mathbf{z}\right) v ; y_{0}, \mathbf{y}\right) .
\end{gathered}
$$


We then give a conceptual construction of toroidal vertex algebras and twisted modules, analogous to a result of [Li2] for vertex algebras. Let $W$ be a vector space and let $r$ and $N$ be positive integers. Set

$\mathcal{E}(W, r ; N)=\operatorname{Hom}\left(W, W\left[\left[x_{1}^{ \pm 1}, \ldots, x_{r}^{ \pm 1}\right]\right]\left(\left(x_{0}^{\frac{1}{N}}\right)\right)\right) \subset \operatorname{End}(W)\left[\left[x_{0}^{ \pm \frac{1}{N}}, x_{1}^{ \pm 1}, \ldots, x_{r}^{ \pm 1}\right]\right]$.

We equip the space $\mathcal{E}(W, r ; N)$ with the following $\mathbb{Z}_{N^{-} \text {grading }}$

$$
\mathcal{E}(W, r ; N)=\bigoplus_{s=0}^{N-1} \mathcal{E}(W, r ; N)_{s}
$$

where $\mathcal{E}(W, r ; N)_{s}=x_{0}^{-\frac{s}{N}} \operatorname{Hom}\left(W, W\left[\left[x_{1}^{ \pm 1}, \ldots, x_{r}^{ \pm 1}\right]\right]\left(\left(x_{0}\right)\right)\right)$. Let $\sigma$ be the linear automorphism of $\mathcal{E}(W, r ; N)$ with $\mathcal{E}(W, r ; N)_{s}$ as the eigenspace of eigenvalue $e^{2 \pi i s / N}$ for $0 \leq s<N$. We call a subset $U$ of $\mathcal{E}(W, r ; N)$ local if for any $a\left(x_{0}, \mathbf{x}\right), b\left(x_{0}, \mathbf{x}\right) \in U$, there exists a nonnegative integer $k$ such that

$$
\left(x_{0}-y_{0}\right)^{k}\left[a\left(x_{0}, \mathbf{x}\right), b\left(y_{0}, \mathbf{y}\right)\right]=0 .
$$

As the main result, we prove that every graded local subspace $U$ of $\mathcal{E}(W, r ; N)$ generates an $(r+1)$-toroidal vertex algebra $\langle U\rangle$ in a certain canonical way with $\sigma$ as an automorphism of period $N$ and that $W$ is a natural $\sigma$-twisted module for $\langle U\rangle$.

The second half of this paper is devoted to establishing an association of toroidal vertex algebras to twisted toroidal Lie algebras. Let $\mathfrak{g}$ be a finite dimensional simple Lie algebra and let $\sigma_{0}, \sigma_{1}, \ldots, \sigma_{r}$ be mutually commuting automorphisms of $\mathfrak{g}$ with finite orders $N_{0}, N_{1}, \ldots, N_{r}$, respectively. Set $G=\left\langle\sigma_{0}, \sigma_{1}, \ldots, \sigma_{r}\right\rangle \subset \operatorname{Aut}(\mathfrak{g})$. Consider the following $(r+1)$-loop Lie algebra

$$
L_{r+1}\left(\mathfrak{g}, N_{0}\right)=\mathfrak{g} \otimes \mathbb{C}\left[t_{0}^{ \pm \frac{1}{N_{0}}}, t_{1}^{ \pm 1}, \ldots, t_{r}^{ \pm 1}\right]
$$

and its central extension

$$
\widehat{L_{r+1}}\left(\mathfrak{g}, N_{0}\right)=\mathfrak{g} \otimes \mathbb{C}\left[t_{0}^{ \pm \frac{1}{N_{0}}}, t_{1}^{ \pm 1}, \ldots, t_{r}^{ \pm 1}\right] \oplus \mathbb{C} \mathfrak{c}
$$

Let $\tau$ be the $G$-fixed points subalgebra

$$
\tau=\left(\widehat{L_{r+1}}\left(\mathfrak{g}, N_{0}\right)\right)^{G}
$$

Note that it was proved in $\mathrm{ABFP}$ that for almost all extended affine Lie algebras of nullity $r+1$, the centerless core is isomorphic to such a Lie algebra $\tau$.

To associate $(r+1)$-toroidal vertex algebras to twisted toroidal Lie algebra $\tau$, we consider a subalgebra of the $(r+1)$-toroidal Lie algebra $\widehat{L_{r+1}}(\mathfrak{g})=\widehat{L_{r+1}}(\mathfrak{g}, 1)$. Set

$$
\mathcal{L}=\left(\bigoplus_{\mathbf{m} \in \mathbb{Z}^{r}} \mathfrak{g}_{\mathbf{m}} \otimes \mathbf{t}^{\mathbf{m}} \mathbb{C}\left[t_{0}^{ \pm 1}\right]\right) \bigoplus \mathbb{C} \mathfrak{c}
$$


where $\mathfrak{g}_{\mathbf{m}}=\left\{a \in \mathfrak{g} \mid \sigma_{j}(a)=\omega_{N_{j}}^{m_{j}} a\right.$ for $\left.1 \leq j \leq r\right\}$ for $\mathbf{m}=\left(m_{1}, \ldots, m_{r}\right) \in \mathbb{Z}^{r}$. Set

$$
\mathcal{L}^{\geq 0}=\left(\bigoplus_{\mathbf{m} \in \mathbb{Z}^{r}} \mathfrak{g}_{\mathbf{m}} \otimes \mathbf{t}^{\mathbf{m}} \mathbb{C}\left[t_{0}\right]\right) \bigoplus \mathbb{C} \mathfrak{c}
$$

a subalgebra of $\mathcal{L}$. For any complex number $\ell$, we construct an $(r+1)$-toroidal vertex algebra $V_{\mathcal{L}}(\ell, 0)$ whose underlying vector space is the following induced module

$$
V_{\mathcal{L}}(\ell, 0)=U(\mathcal{L}) \otimes_{U(\mathcal{L} \geq 0)}(\mathfrak{g}+\mathbb{C})
$$

where $\mathfrak{g} \oplus \mathbb{C}$ is equipped with a suitably defined $\mathcal{L}^{\geq 0}$-module structure such that $\mathfrak{c}$ acts as scalar $\ell$. The automorphism $\sigma_{0}$ of $\mathfrak{g}$ is shown to induce an automorphism $\tilde{\sigma}$ of $V_{\mathcal{L}}(\ell, 0)$ with order $N_{0}$. Then we show that the category of restricted $\tau$-modules of level $\ell$ is naturally isomorphic to that of $\tilde{\sigma}$-twisted $V_{\mathcal{L}}(\ell, 0)$-modules satisfying a certain equivariance property (see Theorem 4.7 for details).

This paper is organized as follows: In Section 2, we define the notation of twisted module for a general toroidal vertex algebra and we present some basic results. In Section 3, we give a general construction of toroidal vertex algebras and their twisted modules. In Section 4, we associate toroidal vertex algebras and their twisted modules to twisted toroidal Lie algebras.

\section{Twisted modules for toroidal vertex algebras}

In this section, we define the notion of twisted module for a toroidal vertex algebra with a finite order automorphism and we present some basic properties for twisted modules.

First of all, throughout this paper, we denote by $\mathbb{C}, \mathbb{N}$, and $\mathbb{Z}$ the field of complex numbers, the set of nonnegative integers, the set of integers, respectively. The symbols $x, y, z, x_{0}, y_{0}, z_{0}, x_{1}, y_{1}, z_{1}, \ldots$ denote mutually commuting independent formal variables. All vector spaces in this paper are considered to be over $\mathbb{C}$.

Let $r$ be a positive integer which is fixed throughout this paper. For any $\mathbf{m}=$ $\left(m_{1}, \ldots, m_{r}\right) \in \mathbb{Z}^{r}$, we set

$$
\mathbf{x}^{\mathbf{m}}=x_{1}^{m_{1}} \cdots x_{r}^{m_{r}}
$$

As a convention, we write

$$
\mathbf{x}^{-1}=x_{1}^{-1} \cdots x_{r}^{-1}, \quad \mathbf{x}^{\mathbf{m}-1}=x_{1}^{m_{1}-1} \cdots x_{r}^{m_{r}-1},
$$

and

$$
\operatorname{Res}_{\mathbf{x}}=\operatorname{Res}_{x_{1}} \cdots \operatorname{Res}_{x_{r}} .
$$


For a vector space $W$ and a positive integer $N$, we set

$$
\mathcal{E}(W, r ; N)=\operatorname{Hom}\left(W, W\left[\left[x_{1}^{ \pm 1}, \ldots, x_{r}^{ \pm 1}\right]\right]\left(\left(x_{0}^{\frac{1}{N}}\right)\right)\right) .
$$

In particular, we write $\mathcal{E}(W, r)$ for $\mathcal{E}(W, r ; 1)$, i.e.,

$$
\mathcal{E}(W, r)=\operatorname{Hom}\left(W, W\left[\left[x_{1}^{ \pm 1}, \ldots, x_{r}^{ \pm 1}\right]\right]\left(\left(x_{0}\right)\right)\right) .
$$

Recall the formal delta-function

$$
\delta(z)=\sum_{n \in \mathbb{Z}} z^{n} \in \mathbb{C}\left[\left[z, z^{-1}\right]\right] .
$$

We have

$$
\delta\left(\frac{z_{1}-z_{2}}{z_{0}}\right)=\sum_{n \in \mathbb{Z}} z_{0}^{-n}\left(z_{1}-z_{2}\right)^{n}=\sum_{n \in \mathbb{Z}} \sum_{i \in \mathbb{N}}(-1)^{i}\left(\begin{array}{c}
n \\
i
\end{array}\right) z_{0}^{-n} z_{1}^{n-i} z_{2}^{i} .
$$

The following is one of the basic properties of the delta-function

$$
z_{0}^{-1} \delta\left(\frac{z_{1}-z_{2}}{z_{0}}\right)\left(\frac{z_{1}-z_{2}}{z_{0}}\right)^{\alpha}=z_{1}^{-1} \delta\left(\frac{z_{0}+z_{2}}{z_{1}}\right)\left(\frac{z_{0}+z_{2}}{z_{1}}\right)^{-\alpha}
$$

for $\alpha \in \mathbb{C}($ see $[\mathrm{DL}])$.

Next, we recall the definition of an $(r+1)$-toroidal vertex algebra from [LTW2].

Definition 2.1. An $(r+1)$-toroidal vertex algebra is a vector space $V$ equipped with a linear map

$$
\begin{aligned}
Y\left(\cdot ; x_{0}, \mathbf{x}\right): \quad & V \rightarrow \mathcal{E}(V, r), \\
v & \mapsto Y\left(v ; x_{0}, \mathbf{x}\right)=\sum_{\left(m_{0}, \mathbf{m}\right) \in \mathbb{Z} \times \mathbb{Z}^{r}} v_{m_{0}, \mathbf{m}} x_{0}^{-m_{0}-1} \mathbf{x}^{-\mathbf{m}}
\end{aligned}
$$

and equipped with a distinguished vector $1 \in V$, satisfying the conditions that

$$
Y\left(\mathbf{1} ; x_{0}, \mathbf{x}\right) v=v \text { and } Y\left(v ; x_{0}, \mathbf{x}\right) \mathbf{1} \in V\left[\left[x_{0}, x_{1}^{ \pm 1}, \ldots, x_{r}^{ \pm 1}\right]\right] \quad \text { for } v \in V
$$

and that for $u, v \in V$,

$$
\begin{gathered}
z_{0}^{-1} \delta\left(\frac{x_{0}-y_{0}}{z_{0}}\right) Y\left(u ; x_{0}, \mathbf{z y}\right) Y\left(v ; y_{0}, \mathbf{y}\right)-z_{0}^{-1} \delta\left(\frac{y_{0}-x_{0}}{-z_{0}}\right) Y\left(v ; y_{0}, \mathbf{y}\right) Y\left(u ; x_{0}, \mathbf{z y}\right) \\
=y_{0}^{-1} \delta\left(\frac{x_{0}-z_{0}}{y_{0}}\right) Y\left(Y\left(u ; z_{0}, \mathbf{z}\right) v ; y_{0}, \mathbf{y}\right)
\end{gathered}
$$

where

$$
Y\left(u ; x_{0}, \mathbf{z y}\right)=\sum_{\left(m_{0}, \mathbf{m}\right) \in \mathbb{Z} \times \mathbb{Z}^{r}} u_{m_{0}, \mathbf{m}} x_{0}^{-m_{0}-1} \mathbf{z}^{-\mathbf{m}} \mathbf{y}^{-\mathbf{m}} .
$$


Let $u \in V$. We define $Y\left(u ; m_{0}, \mathbf{x}\right)$ and $Y\left(u ; x_{0}, \mathbf{m}\right)$ for $m_{0} \in \mathbb{Z}, \mathbf{m} \in \mathbb{Z}^{r}$ by

$$
Y\left(u ; x_{0}, \mathbf{x}\right)=\sum_{m_{0} \in \mathbb{Z}} Y\left(u ; m_{0}, \mathbf{x}\right) x_{0}^{-m_{0}-1}=\sum_{\mathbf{m} \in \mathbb{Z}^{r}} Y\left(u ; x_{0}, \mathbf{m}\right) \mathbf{x}^{-\mathbf{m}} .
$$

From the Jacobi identity (2.4) we get

$$
\begin{aligned}
& {\left[Y\left(u ; x_{0}, \mathbf{z y}\right), Y\left(v, y_{0}, \mathbf{y}\right)\right] } \\
= & \operatorname{Res}_{z_{0}} \sum_{j \geq 0} \frac{1}{j !}\left(\frac{\partial}{\partial y_{0}}\right)^{j}\left(x_{0}^{-1} \delta\left(\frac{y_{0}}{x_{0}}\right)\right) z_{0}^{j} Y\left(Y\left(u ; z_{0}, \mathbf{z}\right) v ; y_{0}, \mathbf{y}\right) \\
= & \sum_{j \geq 0} \frac{1}{j !}\left(\frac{\partial}{\partial y_{0}}\right)^{j}\left(x_{0}^{-1} \delta\left(\frac{y_{0}}{x_{0}}\right)\right) Y\left(Y(u ; j, \mathbf{z}) v ; y_{0}, \mathbf{y}\right) .
\end{aligned}
$$

Furthermore, we have

$$
\left[Y\left(u ; x_{0}, \mathbf{m}\right), Y\left(v ; y_{0}, \mathbf{y}\right)\right]=\mathbf{y}^{\mathbf{m}} \sum_{j \geq 0} Y\left(u_{j, \mathbf{m}} v ; y_{0}, \mathbf{y}\right) \frac{1}{j !}\left(\frac{\partial}{\partial y_{0}}\right)^{j} x_{0}^{-1} \delta\left(\frac{y_{0}}{x_{0}}\right)
$$

for $\mathbf{m} \in \mathbb{Z}^{r}$.

Let $V_{1}$ and $V_{2}$ be $(r+1)$-toroidal vertex algebras. An $(r+1)$-toroidal vertex algebra homomorphism from $V_{1}$ to $V_{2}$ is a linear map $\sigma$ such that

$$
\sigma(\mathbf{1})=\mathbf{1} \text { and } \sigma\left(Y\left(u ; x_{0}, \mathbf{x}\right) v\right)=Y\left(\sigma(u) ; x_{0}, \mathbf{x}\right) \sigma(v) \quad \text { for } u, v \in V_{1} .
$$

An automorphism of an $(r+1)$-toroidal vertex algebra $V$ is defined to be a bijective homomorphism from $V$ to $V$.

Next, we define a notion of twisted module for an $(r+1)$-toroidal vertex algebra $V$. Let $\sigma$ be a finite order automorphism of $V$ and let $N$ be a period of $\sigma$. (Here, $N$ is a positive integer such that $\sigma^{N}=1$, but $N$ is not necessarily the order of $\sigma$.) Set

$$
\omega_{N}=\exp (2 \pi \sqrt{-1} / N)
$$

the principal primitive $N$-th root of unity. Then $V=\oplus_{s=0}^{N-1} V^{s}$, where $V^{j}=$ $\left\{u \in V \mid \sigma(u)=\omega_{N}^{j} u\right\}$ for any $j \in \mathbb{Z}$.

Definition 2.2. A $\sigma$-twisted $V$-module is a vector space $W$ equipped with a linear map

$$
\begin{aligned}
Y_{W}\left(\cdot ; x_{0}, \mathbf{x}\right): \quad & V \rightarrow \mathcal{E}(W, r ; N) \\
v & \mapsto Y_{W}\left(v ; x_{0}, \mathbf{x}\right)=\sum_{\left(m_{0}, \mathbf{m}\right) \in \frac{1}{N} \mathbb{Z} \times \mathbb{Z}^{r}} v_{m_{0}, \mathbf{m}} x_{0}^{-m_{0}-1} \mathbf{x}^{-\mathbf{m}},
\end{aligned}
$$

such that

$$
Y_{W}\left(\mathbf{1} ; x_{0}, \mathbf{x}\right)=1_{W}
$$


and such that the following $\sigma$-twisted Jacobi identity holds for $u, v \in V$ :

$$
\begin{gathered}
z_{0}^{-1} \delta\left(\frac{x_{0}-y_{0}}{z_{0}}\right) Y_{W}\left(u ; x_{0}, \mathbf{z y}\right) Y_{W}\left(v ; y_{0}, \mathbf{y}\right)-z_{0}^{-1} \delta\left(\frac{y_{0}-x_{0}}{-z_{0}}\right) Y_{W}\left(v ; y_{0}, \mathbf{y}\right) Y_{W}\left(u ; x_{0}, \mathbf{z y}\right) \\
\quad=\frac{1}{N} \sum_{j=0}^{N-1} y_{0}^{-1} \delta\left(\omega_{N}^{j}\left(\frac{x_{0}-z_{0}}{y_{0}}\right)^{\frac{1}{N}}\right) Y_{W}\left(Y\left(\sigma^{j} u ; z_{0}, \mathbf{z}\right) v ; y_{0}, \mathbf{y}\right) .
\end{gathered}
$$

Lemma 2.3. Let $\left(W, Y_{W}\right)$ be a $\sigma$-twisted $V$-module and let $u, v \in V$. Assume $u \in V^{s}$ with $0 \leq s<N$. Then

$$
Y_{W}\left(u ; x_{0}, \mathbf{x}\right) \in x_{0}^{-\frac{s}{N}} \mathcal{E}(W, r) .
$$

Proof. As $u \in V^{s}$, from (2.8) we get

$$
\begin{aligned}
& z_{0}^{-1} \delta\left(\frac{x_{0}-y_{0}}{z_{0}}\right) Y_{W}\left(u ; x_{0}, \mathbf{z y}\right) Y_{W}\left(v ; y_{0}, \mathbf{y}\right)-z_{0}^{-1} \delta\left(\frac{y_{0}-x_{0}}{-z_{0}}\right) Y_{W}\left(v ; y_{0}, \mathbf{y}\right) Y_{W}\left(u ; x_{0}, \mathbf{z y}\right) \\
& \quad=y_{0}^{-1} \delta\left(\frac{x_{0}-z_{0}}{y_{0}}\right)\left(\frac{x_{0}-z_{0}}{y_{0}}\right)^{-\frac{s}{N}} Y_{W}\left(Y\left(u ; z_{0}, \mathbf{z}\right) v ; y_{0}, \mathbf{y}\right)
\end{aligned}
$$

Using (2.3), one can also write (2.10) as

$$
\begin{aligned}
z_{0}^{-1} \delta & \left(\frac{x_{0}-y_{0}}{z_{0}}\right) Y_{W}\left(u ; x_{0}, \mathbf{z y}\right) Y_{W}\left(v ; y_{0}, \mathbf{y}\right)-z_{0}^{-1} \delta\left(\frac{y_{0}-x_{0}}{-z_{0}}\right) Y_{W}\left(v ; y_{0}, \mathbf{y}\right) Y_{W}\left(u ; x_{0}, \mathbf{z y}\right) \\
& =x_{0}^{-1} \delta\left(\frac{y_{0}+z_{0}}{x_{0}}\right)\left(\frac{y_{0}+z_{0}}{x_{0}}\right)^{\frac{s}{N}} Y_{W}\left(Y\left(u ; z_{0}, \mathbf{z}\right) v ; y_{0}, \mathbf{y}\right)
\end{aligned}
$$

Taking $v=\mathbf{1}$ in (2.10), we get

$$
\begin{aligned}
& y_{0}^{-1} \delta\left(\frac{x_{0}-z_{0}}{y_{0}}\right) Y_{W}\left(u ; x_{0}, \mathbf{z y}\right) \\
= & y_{0}^{-1} \delta\left(\frac{x_{0}-z_{0}}{y_{0}}\right)\left(\frac{x_{0}-z_{0}}{y_{0}}\right)^{-\frac{s}{N}} Y_{W}\left(Y\left(u ; z_{0}, \mathbf{z}\right) \mathbf{1} ; y_{0}, \mathbf{y}\right) .
\end{aligned}
$$

Then applying $\operatorname{Res}_{z_{0}} z_{0}^{-1}$ (or setting $z_{0}=0$ ) we have

$$
y_{0}^{-1} \delta\left(\frac{x_{0}}{y_{0}}\right) Y_{W}\left(u ; x_{0}, \mathbf{z y}\right)=y_{0}^{-1} \delta\left(\frac{x_{0}}{y_{0}}\right)\left(\frac{x_{0}}{y_{0}}\right)^{-\frac{s}{N}} \sum_{\mathbf{n} \in \mathbb{Z}^{r}} Y_{W}\left(u_{-1, \mathbf{n}} \mathbf{1} ; y_{0}, \mathbf{y}\right) \mathbf{z}^{-\mathbf{n}} \text {. }
$$

From this we get (2.9).

Let $\left(W, Y_{W}\right)$ be a $\sigma$-twisted $V$-module and let $u \in V^{s}, v \in V$ as in Lemma 2.3. Applying $\operatorname{Res}_{z_{0}}$ to (2.11), we obtain the following twisted commutator formula

$$
\begin{aligned}
& {\left[Y_{W}\left(u ; x_{0}, \mathbf{z y}\right), Y_{W}\left(v, y_{0}, \mathbf{y}\right)\right] } \\
= & \operatorname{Res}_{z_{0}} \sum_{j \geq 0} \frac{1}{j !}\left(\frac{\partial}{\partial y_{0}}\right)^{j}\left(x_{0}^{-1} \delta\left(\frac{y_{0}}{x_{0}}\right)\left(\frac{y_{0}}{x_{0}}\right)^{\frac{s}{N}}\right) z_{0}^{j} Y_{W}\left(Y\left(u ; z_{0}, \mathbf{z}\right) v ; y_{0}, \mathbf{y}\right) \\
= & \sum_{j \geq 0} \frac{1}{j !}\left(\frac{\partial}{\partial y_{0}}\right)^{j}\left(x_{0}^{-1} \delta\left(\frac{y_{0}}{x_{0}}\right)\left(\frac{y_{0}}{x_{0}}\right)^{\frac{s}{N}}\right) Y_{W}\left(Y(u ; j, \mathbf{z}) v ; y_{0}, \mathbf{y}\right) .
\end{aligned}
$$


Furthermore, we have

$$
\begin{aligned}
& {\left[Y_{W}\left(u ; x_{0}, \mathbf{m}\right), Y_{W}\left(v, y_{0}, \mathbf{y}\right)\right] } \\
= & \mathbf{y}^{\mathbf{m}} \sum_{j \geq 0} \frac{1}{j !}\left(\frac{\partial}{\partial y_{0}}\right)^{j}\left(x_{0}^{-1} \delta\left(\frac{y_{0}}{x_{0}}\right)\left(\frac{y_{0}}{x_{0}}\right)^{\frac{s}{N}}\right) Y_{W}\left(u_{j, \mathbf{m}} v ; y_{0}, \mathbf{y}\right)
\end{aligned}
$$

for $\mathbf{m} \in \mathbb{Z}^{r}$, where

$$
Y_{W}\left(u ; x_{0}, \mathbf{x}\right)=\sum_{\mathbf{m} \in \mathbb{Z}^{r}} Y_{W}\left(u ; x_{0}, \mathbf{m}\right) \mathbf{x}^{-\mathbf{m}} .
$$

Multiplying (2.10) by $\left(\frac{x_{0}-z_{0}}{y_{0}}\right)^{\frac{s}{N}}$, then applying $\operatorname{Res}_{x_{0}}$, we get a twisted iterate formula

$$
Y_{W}\left(Y\left(u ; z_{0}, \mathbf{z}\right) v ; y_{0}, \mathbf{y}\right)=\operatorname{Res}_{x_{0}}\left(\frac{x_{0}-z_{0}}{y_{0}}\right)^{\frac{s}{N}} \cdot X
$$

where

$$
X=z_{0}^{-1} \delta\left(\frac{x_{0}-y_{0}}{z_{0}}\right) Y_{W}\left(u ; x_{0}, \mathbf{z y}\right) Y_{W}\left(v ; y_{0}, \mathbf{y}\right)-z_{0}^{-1} \delta\left(\frac{y_{0}-x_{0}}{-z_{0}}\right) Y_{W}\left(v ; y_{0}, \mathbf{y}\right) Y_{W}\left(u ; x_{0}, \mathbf{z y}\right) .
$$

If we use (2.11), similarly we get the following variation

$$
Y_{W}\left(Y\left(u ; z_{0}, \mathbf{z}\right) v ; y_{0}, \mathbf{y}\right)=\operatorname{Res}_{x_{0}}\left(\frac{y_{0}+z_{0}}{x_{0}}\right)^{-\frac{s}{N}} \cdot X,
$$

where $X$ is given as above.

Lemma 2.4. Let $\left(W, Y_{W}\right)$ be a $\sigma$-twisted $V$-module. (a) For $u, v \in V$, there exists a nonnegative integer $k$ such that

$$
\left(x_{0}-y_{0}\right)^{k}\left[Y_{W}\left(u ; x_{0}, \mathbf{x}\right), Y_{W}\left(v ; y_{0}, \mathbf{y}\right)\right]=0 .
$$

(b) For $u \in V^{s}, v \in V, w \in W$, there exists a nonnegative integer $\ell$ such that

$$
\begin{aligned}
& \left(z_{0}+y_{0}\right)^{\ell+\frac{s}{N}} Y_{W}\left(u ; z_{0}+y_{0}, \mathbf{z y}\right) Y_{W}\left(v ; y_{0}, \mathbf{y}\right) w \\
= & \left(y_{0}+z_{0}\right)^{\ell+\frac{s}{N}} Y_{W}\left(Y\left(u ; z_{0}, \mathbf{z}\right) v ; y_{0}, \mathbf{y}\right) w .
\end{aligned}
$$

Proof. For part (a), let $k \in \mathbb{N}$ be such that $z_{0}^{k} Y\left(u ; z_{0}, \mathbf{z}\right) v \in V\left[\left[z_{0}, \mathbf{z}, \mathbf{z}^{-1}\right]\right]$. Then the assertion follows from (2.12) immediately.

To prove part (b), let $k \in \mathbb{N}$ be such that (2.16) holds and let $l \in \mathbb{N}$ be such that

$$
x_{0}^{l+\frac{s}{N}} Y_{W}\left(u ; x_{0}, \mathbf{z y}\right) w \in W\left[\left[x_{0}, \mathbf{y}, \mathbf{y}^{-1}, \mathbf{z}, \mathbf{z}^{-1}\right]\right] .
$$

Then 
$x_{0}^{l+\frac{s}{N}}\left(x_{0}-y_{0}\right)^{k} Y_{W}\left(u ; x_{0}, \mathbf{z y}\right) Y_{W}\left(v ; y_{0}, \mathbf{y}\right) w=x_{0}^{l+\frac{s}{N}}\left(x_{0}-y_{0}\right)^{k} Y_{W}\left(v ; y_{0}, \mathbf{y}\right) Y_{W}\left(u ; x_{0}, \mathbf{z y}\right) w$

and

$$
x_{0}^{l+\frac{s}{N}}\left(x_{0}-y_{0}\right)^{k} Y_{W}\left(v ; y_{0}, \mathbf{y}\right) Y_{W}\left(u ; x_{0}, \mathbf{z y}\right) w \in W\left[\left[x_{0}, y_{0}^{ \pm 1}, \mathbf{y}, \mathbf{y}^{-1}, \mathbf{z}, \mathbf{z}^{-1}\right]\right],
$$

which imply

$$
x_{0}^{l+\frac{s}{N}}\left(x_{0}-y_{0}\right)^{k} Y_{W}\left(u ; x_{0}, \mathbf{z y}\right) Y_{W}\left(v ; y_{0}, \mathbf{y}\right) w \in W\left[\left[x_{0}, y_{0}^{ \pm 1}, \mathbf{y}, \mathbf{y}^{-1}, \mathbf{z}, \mathbf{z}^{-1}\right]\right] .
$$

In view of this, we have

$$
\begin{aligned}
& {\left.\left[\left(x_{0}-y_{0}\right)^{k} x_{0}^{l+\frac{s}{N}} Y_{W}\left(u ; x_{0}, \mathbf{z y}\right) Y_{W}\left(v ; y_{0}, \mathbf{y}\right) w\right]\right|_{x_{0}=y_{0}+z_{0}} } \\
= & {\left.\left[\left(x_{0}-y_{0}\right)^{k} x_{0}^{l+\frac{s}{N}} Y_{W}\left(u ; x_{0}, \mathbf{z y}\right) Y_{W}\left(v ; y_{0}, \mathbf{y}\right) w\right]\right|_{x_{0}=z_{0}+y_{0}} . }
\end{aligned}
$$

Using (2.15), delta-function substitution, and (2.16), we obtain

$$
\begin{aligned}
& z_{0}^{k}\left(y_{0}+z_{0}\right)^{l+\frac{s}{N}} Y_{W}\left(Y\left(u ; z_{0}, \mathbf{z}\right) v ; y_{0}, \mathbf{y}\right) w \\
= & \operatorname{Res}_{x_{0}} x_{0}^{-1} \delta\left(\frac{y_{0}+z_{0}}{x_{0}}\right)\left(\frac{y_{0}+z_{0}}{x_{0}}\right)^{-\frac{s}{N}}\left(y_{0}+z_{0}\right)^{l+\frac{s}{N}} \\
& \cdot\left[\left(x_{0}-y_{0}\right)^{k} Y_{W}\left(u ; x_{0}, \mathbf{z y}\right) Y_{W}\left(v ; y_{0}, \mathbf{y}\right) w\right] \\
= & \operatorname{Res}_{x_{0}} x_{0}^{-1} \delta\left(\frac{y_{0}+z_{0}}{x_{0}}\right)\left[\left(x_{0}-y_{0}\right)^{k} x_{0}^{l+\frac{s}{N}} Y_{W}\left(u ; x_{0}, \mathbf{z y}\right) Y_{W}\left(v ; y_{0}, \mathbf{y}\right) w\right] \\
= & {\left.\left[\left(x_{0}-y_{0}\right)^{k} x_{0}^{l+\frac{s}{N}} Y_{W}\left(u ; x_{0}, \mathbf{z y}\right) Y_{W}\left(v ; y_{0}, \mathbf{y}\right) w\right]\right|_{x_{0}=y_{0}+z_{0}} } \\
= & {\left.\left[\left(x_{0}-y_{0}\right)^{k} x_{0}^{l+\frac{s}{N}} Y_{W}\left(u ; x_{0}, \mathbf{z y}\right) Y_{W}\left(v ; y_{0}, \mathbf{y}\right) w\right]\right|_{x_{0}=z_{0}+y_{0}} } \\
= & z_{0}^{k}\left(z_{0}+y_{0}\right)^{l+\frac{s}{N}} Y_{W}\left(u ; z_{0}+y_{0}, \mathbf{z y}\right) Y_{W}\left(v ; y_{0}, \mathbf{y}\right) w .
\end{aligned}
$$

Multiplying both sides by $z_{0}^{-k}$, we obtain (2.17).

The property (a) in Lemma 2.4 is called weak commutativity, while property (b) is called weak twisted associativity. Just as with twisted modules for vertex algebras (see [Li2]), the converse of Lemma 2.4 also holds.

Lemma 2.5. Let $V$ be an $(r+1)$-toroidal vertex algebra and let $\sigma$ be an automorphism of period $N$. In the definition of a $\sigma$-twisted $V$-module, the twisted Jacobi identity can be equivalently replaced by the weak commutativity and the twisted weak associativity.

Proof. Let $u \in V^{s}, v \in V$ and $w \in W$ with $0 \leq s<N$. Let $k \in \mathbb{N}$ be such that (2.16) and (2.17) hold and such that $x_{0}^{k+\frac{s}{N}} Y_{W}\left(u ; x_{0}, \mathbf{x}\right) w \in W\left[\left[x_{0}, \mathbf{x}, \mathbf{x}^{-1}\right]\right]$. Then $\left(x_{0}-y_{0}\right)^{k} x_{0}^{k+\frac{s}{N}} Y_{W}\left(u ; x_{0}, \mathbf{z y}\right) Y_{W}\left(v ; y_{0}, \mathbf{y}\right) w=\left(x_{0}-y_{0}\right)^{k} x_{0}^{k+\frac{s}{N}} Y_{W}\left(v ; y_{0}, \mathbf{y}\right) Y_{W}\left(u ; x_{0}, \mathbf{z y}\right) w$. 
As the expression on the right-hand side involves only nonnegative integer powers of $x_{0}$, so does the expression on the left-hand side. Thus

$$
\begin{aligned}
& {\left.\left[\left(x_{0}-y_{0}\right)^{k} x_{0}^{k+\frac{s}{N}} Y_{W}\left(u ; x_{0}, \mathbf{z y}\right) Y_{W}\left(v ; y_{0}, \mathbf{y}\right) w\right]\right|_{x_{0}=y_{0}+z_{0}} } \\
= & {\left.\left[\left(x_{0}-y_{0}\right)^{k} x_{0}^{k+\frac{s}{N}} Y_{W}\left(u ; x_{0}, \mathbf{z y}\right) Y_{W}\left(v ; y_{0}, \mathbf{y}\right) w\right]\right|_{x_{0}=z_{0}+y_{0}} . }
\end{aligned}
$$

Then using delta-function substitution and (2.17) we get

$$
\begin{aligned}
& z_{0}^{k} x_{0}^{k} z_{0}^{-1} \delta\left(\frac{x_{0}-y_{0}}{z_{0}}\right) Y_{W}\left(u ; x_{0}, \mathbf{z y}\right) Y_{W}\left(v ; y_{0}, \mathbf{y}\right) w \\
& -z_{0}^{k} x_{0}^{k} z_{0}^{-1} \delta\left(\frac{y_{0}-x_{0}}{-z_{0}}\right) Y_{W}\left(v ; y_{0}, \mathbf{y}\right) Y_{W}\left(u ; x_{0}, \mathbf{z y}\right) w \\
& =z_{0}^{-1} \delta\left(\frac{x_{0}-y_{0}}{z_{0}}\right) x_{0}^{-\frac{s}{N}}\left[\left(x_{0}-y_{0}\right)^{k} x_{0}^{k+\frac{s}{N}} Y_{W}\left(u ; x_{0}, \mathbf{z y}\right) Y_{W}\left(v ; y_{0}, \mathbf{y}\right) w\right] \\
& -z_{0}^{-1} \delta\left(\frac{y_{0}-x_{0}}{-z_{0}}\right) x_{0}^{-\frac{s}{N}}\left[\left(x_{0}-y_{0}\right)^{k} x_{0}^{k+\frac{s}{N}} Y_{W}\left(v ; y_{0}, \mathbf{y}\right) Y_{W}\left(u ; x_{0}, \mathbf{z y}\right) w\right] \\
& =y_{0}^{-1} \delta\left(\frac{x_{0}-z_{0}}{y_{0}}\right) x_{0}^{-\frac{s}{N}}\left[\left(x_{0}-y_{0}\right)^{k} x_{0}^{k+\frac{s}{N}} Y_{W}\left(u ; x_{0}, \mathbf{z y}\right) Y_{W}\left(v ; y_{0}, \mathbf{y}\right) w\right] \\
& =\left.x_{0}^{-1} \delta\left(\frac{y_{0}+z_{0}}{x_{0}}\right) x_{0}^{-\frac{s}{N}}\left[\left(x_{0}-y_{0}\right)^{k} x_{0}^{k+\frac{s}{N}} Y_{W}\left(u ; x_{0}, \mathbf{z y}\right) Y_{W}\left(v ; y_{0}, \mathbf{y}\right) w\right]\right|_{x_{0}=y_{0}+z_{0}} \\
& =\left.x_{0}^{-1} \delta\left(\frac{y_{0}+z_{0}}{x_{0}}\right) x_{0}^{-\frac{s}{N}}\left[\left(x_{0}-y_{0}\right)^{k} x_{0}^{k+\frac{s}{N}} Y_{W}\left(u ; x_{0}, \mathbf{z y}\right) Y_{W}\left(v ; y_{0}, \mathbf{y}\right) w\right]\right|_{x_{0}=z_{0}+y_{0}} \\
& =x_{0}^{-1} \delta\left(\frac{y_{0}+z_{0}}{x_{0}}\right) x_{0}^{-\frac{s}{N}} z_{0}^{k}\left(z_{0}+y_{0}\right)^{k+\frac{s}{N}} Y_{W}\left(u ; z_{0}+y_{0}, \mathbf{z y}\right) Y_{W}\left(v ; y_{0}, \mathbf{y}\right) w \\
& =x_{0}^{-1} \delta\left(\frac{y_{0}+z_{0}}{x_{0}}\right) x_{0}^{-\frac{s}{N}} z_{0}^{k}\left(y_{0}+z_{0}\right)^{k+\frac{s}{N}} Y_{W}\left(Y\left(u ; z_{0}, z\right) v ; y_{0}, \mathbf{y}\right) w \\
& =x_{0}^{k} z_{0}^{k} x_{0}^{-1} \delta\left(\frac{y_{0}+z_{0}}{x_{0}}\right)\left(\frac{y_{0}+z_{0}}{x_{0}}\right)^{\frac{s}{N}} Y_{W}\left(Y\left(u ; z_{0}, z\right) v ; y_{0}, \mathbf{y}\right) w \\
& \left.=x_{0}^{k} z_{0}^{k} y_{0}^{-1} \delta\left(\frac{x_{0}-z_{0}}{y_{0}}\right)\left(\frac{x_{0}-z_{0}}{y_{0}}\right)^{-\frac{s}{N}} Y_{W}\left(Y\left(u ; z_{0}, z\right) v ; y_{0}, \mathbf{y}\right)\right) w .
\end{aligned}
$$

Multiplying by $x_{0}^{-k} z_{0}^{-k}$, we obtain the twisted Jacobi identity.

Using (2.13), Lemma 2.3 in [Li2], and (2.7), we immediately have the following analogue of one half of Lemma 2.11 therein:

Lemma 2.6. Let $V$ be an $(r+1)$-toroidal vertex algebra with a finite order automorphism $\sigma$ of period $N$ and let $\left(W, Y_{W}\right)$ be a faithful $\sigma$-twisted $V$-module. Let

$$
a, b, c^{(0)}, c^{(1)}, \ldots, c^{(k)} \in V, \mathbf{m} \in \mathbb{Z}^{r}
$$


and assume $a \in V^{s}$ with $0 \leq s<N$. If

$$
\begin{aligned}
& {\left[Y_{W}\left(a ; x_{0}, \mathbf{m}\right), Y_{W}\left(b ; y_{0}, \mathbf{y}\right)\right] } \\
= & \mathbf{y}^{\mathbf{m}} \sum_{j=0}^{k} Y_{W}\left(c^{(j)} ; y_{0}, \mathbf{y}\right) \frac{1}{j !}\left(\frac{\partial}{\partial y_{0}}\right)^{j}\left(x_{0}^{-1} \delta\left(\frac{y_{0}}{x_{0}}\right)\left(\frac{y_{0}}{x_{0}}\right)^{\frac{s}{N}}\right)
\end{aligned}
$$

on $W$, then we have

$$
a_{j, \mathbf{m}} b=c^{(j)} \quad \text { for } 0 \leq j \leq k \text { and } a_{j, \mathbf{m}} b=0 \quad \text { for } j>k
$$

and

$$
\left[Y\left(a ; x_{0}, \mathbf{m}\right), Y\left(b ; y_{0}, \mathbf{y}\right)\right]=\mathbf{y}^{\mathbf{m}} \sum_{j=0}^{k} Y\left(c^{(j)} ; y_{0}, \mathbf{y}\right) \frac{1}{j !}\left(\frac{\partial}{\partial y_{0}}\right)^{j} x_{0}^{-1} \delta\left(\frac{y_{0}}{x_{0}}\right)
$$

on $V$.

Remark 2.7. Note that for twisted modules for a vertex algebra, the converse of Lemma 2.6 is also true (see [Li2]) as a vertex algebra itself is always a faithful module. However, this is not the case here; an $(r+1)$-toroidal vertex algebra itself is not necessarily a faithful module. Due to this, we cannot claim the converse of Lemma 2.6.

\section{Construction of toroidal vertex algebras and their twisted modules}

In this section, we give a general construction of $(r+1)$-toroidal vertex algebras and their twisted modules from local subsets of $\mathcal{E}(W, r ; N)$ with $W$ being an arbitrary vector space.

Let $W$ be a vector space in addition to positive integers $r$ and $N$, which are all fixed throughout this section. Recall

$$
\mathcal{E}(W, r ; N)=\operatorname{Hom}\left(W, W\left[\left[x_{1}^{ \pm 1}, \ldots, x_{r}^{ \pm 1}\right]\right]\left(\left(x_{0}^{\frac{1}{N}}\right)\right)\right) .
$$

We equip $\operatorname{End}(W)\left[\left[x_{0}^{ \pm \frac{1}{N}}, x_{1}^{ \pm 1}, \ldots, x_{r}^{ \pm 1}\right]\right]$ with the following $\mathbb{Z}_{N^{-} \text {grading }}$

$$
\operatorname{End}(W)\left[\left[x_{0}^{ \pm \frac{1}{N}}, x_{1}^{ \pm 1}, \ldots, x_{r}^{ \pm 1}\right]\right]=\oplus_{[j] \in \mathbb{Z}_{N}} x^{-\frac{j}{N}} \operatorname{End}(W)\left[\left[x_{0}^{ \pm 1}, x_{1}^{ \pm 1}, \ldots, x_{r}^{ \pm 1}\right]\right]
$$

Let $\sigma$ be the corresponding linear automorphism of $\operatorname{End}(W)\left[\left[x_{0}^{ \pm \frac{1}{N}}, x_{1}^{ \pm 1}, \ldots, x_{r}^{ \pm 1}\right]\right]$, with $x^{-\frac{j}{N}} \operatorname{End}(W)\left[\left[x_{0}^{ \pm 1}, x_{1}^{ \pm 1}, \ldots, x_{r}^{ \pm 1}\right]\right]$ as the eigenspace of eigenvalue $\omega_{N}^{j}$ for $0 \leq$ $j<N$. Namely,

$$
\sigma\left(f\left(x_{0}^{\frac{1}{N}}, x_{1}, \ldots, x_{r}\right)\right)=f\left(\omega_{N}^{-1} x_{0}^{\frac{1}{N}}, x_{1}, \ldots, x_{r}\right) .
$$


We see that a subspace of $\operatorname{End}(W)\left[\left[x_{0}^{ \pm \frac{1}{N}}, x_{1}^{ \pm 1}, \ldots, x_{r}^{ \pm 1}\right]\right]$ is $\sigma$-stable if and only if it is graded. It is clear that $\mathcal{E}(W, r ; N)$ is a graded subspace, so that $\sigma$ is a linear automorphism. We have

$$
\mathcal{E}(W, r ; N)=\oplus_{0 \leq j \leq N-1} \mathcal{E}(W, r ; N)_{j},
$$

where

$$
\mathcal{E}(W, r ; N)_{j}=x_{0}^{-\frac{j}{N}} \operatorname{Hom}\left(W, W\left[\left[x_{1}^{ \pm 1}, \ldots, x_{r}^{ \pm 1}\right]\right]\left(\left(x_{0}\right)\right)\right)=x_{0}^{-\frac{j}{N}} \mathcal{E}(W, r) .
$$

Let $a\left(x_{0}, \mathbf{x}\right), b\left(x_{0}, \mathbf{x}\right) \in \mathcal{E}(W, r ; N)$. We say that $a\left(x_{0}, \mathbf{x}\right)$ and $b\left(x_{0}, \mathbf{x}\right)$ are mutually local if there exists a nonnegative integer $k$ such that

$$
\left(x_{0}-y_{0}\right)^{k}\left[a\left(x_{0}, \mathbf{x}\right), b\left(y_{0}, \mathbf{y}\right)\right]=0 \text {. }
$$

Furthermore, we say a subset $U$ of $\mathcal{E}(W, r ; N)$ is local if for any $a\left(x_{0}, \mathbf{x}\right), b\left(x_{0}, \mathbf{x}\right) \in U$, $a\left(x_{0}, \mathbf{x}\right)$ and $b\left(x_{0}, \mathbf{x}\right)$ are mutually local.

Definition 3.1. Let $a\left(x_{0}, \mathbf{x}\right), b\left(x_{0}, \mathbf{x}\right) \in \mathcal{E}(W, r ; N)$. Assume that $a\left(x_{0}, \mathbf{x}\right)$ and $b\left(x_{0}, \mathbf{x}\right)$ are mutually local and assume $a\left(x_{0}, \mathbf{x}\right) \in \mathcal{E}(W, r ; N)_{j}$ with $0 \leq j<N$. Then we define

$$
a\left(y_{0}, \mathbf{y}\right)_{m_{0}, \mathbf{m}} b\left(y_{0}, \mathbf{y}\right) \in \mathcal{E}(W, r ; N) \quad \text { for }\left(m_{0}, \mathbf{m}\right) \in \mathbb{Z} \times \mathbb{Z}^{r}
$$

in terms of generating function

$$
Y_{\mathcal{E}}\left(a\left(y_{0}, \mathbf{y}\right) ; z_{0}, \mathbf{z}\right) b\left(y_{0}, \mathbf{y}\right)=\sum_{\left(m_{0}, \mathbf{m}\right) \in \mathbb{Z} \times \mathbb{Z}^{r}} a\left(y_{0}, \mathbf{y}\right)_{m_{0}, \mathbf{m}} b\left(y_{0}, \mathbf{y}\right) z_{0}^{-m_{0}-1} \mathbf{z}^{-\mathbf{m}}
$$

by

$$
Y_{\mathcal{E}}\left(a\left(y_{0}, \mathbf{y}\right) ; z_{0}, \mathbf{z}\right) b\left(y_{0}, \mathbf{y}\right)=\operatorname{Res}_{x_{0}}\left(\frac{x_{0}-z_{0}}{y_{0}}\right)^{\frac{j}{N}} \cdot X
$$

where

$$
X=z_{0}^{-1} \delta\left(\frac{x_{0}-y_{0}}{z_{0}}\right) a\left(x_{0}, \mathbf{z y}\right) b\left(y_{0}, \mathbf{y}\right)-z_{0}^{-1} \delta\left(\frac{y_{0}-x_{0}}{-z_{0}}\right) b\left(y_{0}, \mathbf{y}\right) a\left(x_{0}, \mathbf{z y}\right)
$$

Lemma 3.2. Assume that $a\left(x_{0}, \mathbf{x}\right) \in \mathcal{E}(W, r ; N)_{j}, b\left(x_{0}, \mathbf{x}\right) \in \mathcal{E}(W, r ; N)_{s}$ with $j, s \in$ $\mathbb{Z}$ and that $a\left(x_{0}, \mathbf{x}\right)$ and $b\left(x_{0}, \mathbf{x}\right)$ are mutually local. Then

$$
a\left(y_{0}, \mathbf{y}\right)_{m_{0}, \mathbf{m}} b\left(y_{0}, \mathbf{y}\right) \in \mathcal{E}(W, r ; N)_{j+s}
$$

for all $\left(m_{0}, \mathbf{m}\right) \in \mathbb{Z} \times \mathbb{Z}^{r}$ and $a\left(y_{0}, \mathbf{y}\right)_{m_{0}, \mathbf{m}} b\left(y_{0}, \mathbf{y}\right)=0$ whenever $m_{0} \geq k$, where $k$ is a nonnegative integer such that (3.4) holds. 
Proof. By definition we have

$$
y_{0}^{\frac{j+s}{N}} Y_{\mathcal{E}}\left(a\left(y_{0}, \mathbf{y}\right) ; z_{0}, \mathbf{z}\right) b\left(y_{0}, \mathbf{y}\right)=\operatorname{Res}_{x_{0}}\left(x_{0}-z_{0}\right)^{\frac{j}{N}} \cdot y_{0}^{\frac{s}{N}} X
$$

where $X$ is given as above. Since $y_{0}^{\frac{s}{N}} X$ involves only integer powers of $y_{0}$ (from Lemma 2.3), so does $y_{0}^{\frac{j+s}{N}} Y_{\mathcal{E}}\left(a\left(y_{0}, \mathbf{y}\right) ; z_{0}, \mathbf{z}\right) b\left(y_{0}, \mathbf{y}\right)$. This proves the first assertion. For the second assertion, note that for $m_{0} \in \mathbb{Z}$,

$$
z_{0}^{m_{0}} Y_{\mathcal{E}}\left(a\left(y_{0}, \mathbf{y}\right) ; z_{0}, \mathbf{z}\right) b\left(y_{0}, \mathbf{y}\right)=\operatorname{Res}_{x_{0}} \sum_{i \geq 0}\left(\begin{array}{c}
\frac{j}{N} \\
i
\end{array}\right)(-1)^{i} x_{0}^{\frac{j}{N}-i} y_{0}^{-\frac{j}{N}} \cdot\left(z_{0}^{i+m_{0}} X\right) .
$$

Let $k \in \mathbb{N}$ be such that (3.4) holds. Noticing that for any integer $q$ with $q \geq k$,

$$
\operatorname{Res}_{z_{0}} z_{0}^{q} X=\left(x_{0}-y_{0}\right)^{q} a\left(x_{0}, \mathbf{z y}\right) b\left(y_{0}, \mathbf{y}\right)-\left(x_{0}-y_{0}\right)^{q} b\left(y_{0}, \mathbf{y}\right) a\left(x_{0}, \mathbf{z y}\right)=0,
$$

we have

$$
\operatorname{Res}_{z_{0}} z_{0}^{m_{0}} Y_{\mathcal{E}}\left(a\left(y_{0}, \mathbf{y}\right) ; z_{0}, \mathbf{z}\right) b\left(y_{0}, \mathbf{y}\right)=0
$$

for any $m_{0} \geq k$. Thus, $a\left(x_{0}, \mathbf{x}\right)_{m_{0}, \mathbf{m}} b\left(x_{0}, \mathbf{x}\right)=0$ for any $\left(m_{0}, \mathbf{m}\right) \in \mathbb{Z} \times \mathbb{Z}^{r}$ with $m_{0} \geq k$.

Remark 3.3. Let $a\left(x_{0}, \mathbf{x}\right) \in \mathcal{E}(W, r ; N)_{j}, b\left(x_{0}, \mathbf{x}\right) \in \mathcal{E}(W, r ; N)_{s}$. Suppose that $a\left(x_{0}, \mathbf{x}\right)$ and $b\left(x_{0}, \mathbf{x}\right)$ are mutually local with $k \in \mathbb{N}$ such that (3.4) holds. Then

$$
\left(x_{0}-y_{0}\right)^{k} x_{0}^{\frac{j}{N}} a\left(x_{0}, \mathbf{z y}\right) b\left(y_{0}, \mathbf{y}\right)=\left(x_{0}-y_{0}\right)^{k} x_{0}^{\frac{j}{N}} b\left(y_{0}, \mathbf{y}\right) a\left(x_{0}, \mathbf{z y}\right),
$$

which (by using the information from two sides) implies

$$
\left(x_{0}-y_{0}\right)^{k} x_{0}^{\frac{j}{N}} a\left(x_{0}, \mathbf{z y}\right) b\left(y_{0}, \mathbf{y}\right) \in \operatorname{Hom}\left(W, W\left[\left[\mathbf{y}, \mathbf{y}^{-1}, \mathbf{z}, \mathbf{z}^{-1}\right]\right]\left(\left(x_{0}, y_{0}\right)\right)\right) .
$$

Using delta-function substitution and (3.4), we get

$$
z_{0}^{k} X=\left(x_{0}-y_{0}\right)^{k} X=y_{0}^{-1} \delta\left(\frac{x_{0}-z_{0}}{y_{0}}\right)\left[\left(x_{0}-y_{0}\right)^{k} a\left(x_{0}, \mathbf{z y}\right) b\left(y_{0}, \mathbf{y}\right)\right] .
$$

Then

$$
\begin{aligned}
& z_{0}^{k} Y_{\mathcal{E}}\left(a\left(y_{0}, \mathbf{y}\right) ; z_{0}, \mathbf{z}\right) b\left(y_{0}, \mathbf{y}\right) \\
= & \operatorname{Res}_{x_{0}}\left(\frac{x_{0}-z_{0}}{y_{0}}\right)^{\frac{j}{N}} \cdot z_{0}^{k} X \\
= & \operatorname{Res}_{x_{0}} y_{0}^{-1} \delta\left(\frac{x_{0}-z_{0}}{y_{0}}\right)\left(\frac{x_{0}-z_{0}}{y_{0}}\right)^{\frac{j}{N}}\left[\left(x_{0}-y_{0}\right)^{k} a\left(x_{0}, \mathbf{z y}\right) b\left(y_{0}, \mathbf{y}\right)\right] \\
= & \operatorname{Res}_{x_{0}} x_{0}^{-1} \delta\left(\frac{y_{0}+z_{0}}{x_{0}}\right)\left(\frac{y_{0}+z_{0}}{x_{0}}\right)^{-\frac{j}{N}}\left[\left(x_{0}-y_{0}\right)^{k} a\left(x_{0}, \mathbf{z y}\right) b\left(y_{0}, \mathbf{y}\right)\right] \\
= & \left.\left(y_{0}+z_{0}\right)^{-\frac{j}{N}}\left[\left(x_{0}-y_{0}\right)^{k} x_{0}^{\frac{j}{N}} a\left(x_{0}, \mathbf{z y}\right) b\left(y_{0}, \mathbf{y}\right)\right]\right|_{x_{0}=y_{0}+z_{0} .} .
\end{aligned}
$$


Therefore, we obtain (cf. [LTW1, Lemma 2.9)

$z_{0}^{k}\left(y_{0}+z_{0}\right)^{\frac{j}{N}} Y_{\mathcal{E}}\left(a\left(y_{0}, \mathbf{y}\right) ; z_{0}, \mathbf{z}\right) b\left(y_{0}, \mathbf{y}\right)=\left.\left[\left(x_{0}-y_{0}\right)^{k} x_{0}^{\frac{j}{N}} a\left(x_{0}, \mathbf{z y}\right) b\left(y_{0}, \mathbf{y}\right)\right]\right|_{x_{0}=y_{0}+z_{0}}(3.9)$

This gives a different definition of $Y_{\mathcal{E}}\left(a\left(y_{0}, \mathbf{y}\right) ; z_{0}, \mathbf{z}\right) b\left(y_{0}, \mathbf{y}\right)$.

For any graded local subspace $U$ of $\mathcal{E}(W, r ; N)$, we extend the definition linearly to define $a\left(y_{0}, \mathbf{y}\right)_{m_{0}, \mathbf{m}} b\left(y_{0}, \mathbf{y}\right)$ for any $a\left(x_{0}, \mathbf{x}\right), b\left(x_{0}, \mathbf{x}\right) \in U$.

The following is a key result to the construction:

Lemma 3.4. Assume that $a\left(x_{0}, \mathbf{x}\right), b\left(x_{0}, \mathbf{x}\right), c\left(x_{0}, \mathbf{x}\right) \in \mathcal{E}(W, r ; N)$ are pairwise local. Then for any $\left(m_{0}, \mathbf{m}\right) \in \mathbb{Z} \times \mathbb{Z}^{r}, a\left(x_{0}, \mathbf{x}\right)_{m_{0}, \mathbf{m}} b\left(x_{0}, \mathbf{x}\right)$ and $c\left(x_{0}, \mathbf{x}\right)$ are local.

Proof. Fix $\left(m_{0}, \mathbf{m}\right) \in \mathbb{Z} \times \mathbb{Z}^{r}$. Let $k \in \mathbb{N}$ be such that $k+m_{0}>0$ and

$$
\begin{aligned}
& \left(x_{0}-y_{0}\right)^{k} a\left(x_{0}, \mathbf{x}\right) b\left(y_{0}, \mathbf{y}\right)=\left(x_{0}-y_{0}\right)^{k} b\left(y_{0}, \mathbf{y}\right) a\left(x_{0}, \mathbf{x}\right), \\
& \left(x_{0}-y_{0}\right)^{k} a\left(x_{0}, \mathbf{x}\right) c\left(y_{0}, \mathbf{y}\right)=\left(x_{0}-y_{0}\right)^{k} c\left(y_{0}, \mathbf{y}\right) a\left(x_{0}, \mathbf{x}\right), \\
& \left(x_{0}-y_{0}\right)^{k} b\left(x_{0}, \mathbf{x}\right) c\left(y_{0}, \mathbf{y}\right)=\left(x_{0}-y_{0}\right)^{k} c\left(y_{0}, \mathbf{y}\right) b\left(x_{0}, \mathbf{x}\right) .
\end{aligned}
$$

Assume $a\left(x_{0}, \mathbf{x}\right) \in \mathcal{E}(W, r ; N)_{s}$ with $0 \leq s<N$. We have

$$
\begin{aligned}
& a\left(x_{0}, \mathbf{x}\right)_{m_{0}, \mathbf{m}} b\left(x_{0}, \mathbf{x}\right) \\
= & \operatorname{Res}_{y_{0}} \mathbf{x}^{-\mathbf{m}} \sum_{i=0}^{\infty}\left(\begin{array}{c}
\frac{s}{N} \\
i
\end{array}\right)\left(\frac{y_{0}}{x_{0}}\right)^{\frac{s}{N}} y_{0}^{-i} \cdot(-1)^{i} \cdot T_{i} \\
= & \operatorname{Res}_{y_{0}} \mathbf{x}^{-\mathbf{m}} \sum_{i=0}^{2 k}\left(\begin{array}{c}
\frac{s}{N} \\
i
\end{array}\right)\left(\frac{y_{0}}{x_{0}}\right)^{\frac{s}{N}} y_{0}^{-i} \cdot(-1)^{i} \cdot T_{i},
\end{aligned}
$$

where

$$
T_{i}=\left(y_{0}-x_{0}\right)^{m_{0}+i} a\left(y_{0}, \mathbf{m}\right) b\left(x_{0}, \mathbf{x}\right)-\left(-x_{0}+y_{0}\right)^{m_{0}+i} b\left(x_{0}, \mathbf{x}\right) a\left(y_{0}, \mathbf{m}\right) .
$$

Since

$$
\begin{aligned}
& \left(x_{0}-z_{0}\right)^{4 k}\left(\left(y_{0}-x_{0}\right)^{m_{0}+i} a\left(y_{0}, \mathbf{m}\right) b\left(x_{0}, \mathbf{x}\right) c\left(z_{0}, \mathbf{z}\right)-\left(-x_{0}+y_{0}\right)^{m_{0}+i} b\left(x_{0}, \mathbf{x}\right) a\left(y_{0}, \mathbf{m}\right) c\left(z_{0}, \mathbf{z}\right)\right) \\
= & \sum_{j=0}^{3 k}\left(\begin{array}{c}
3 k \\
j
\end{array}\right)\left(x_{0}-y_{0}\right)^{3 k-j}\left(y_{0}-z_{0}\right)^{j}\left(x_{0}-z_{0}\right)^{k} . \\
= & \cdot\left(\left(y_{0}-x_{0}\right)^{m_{0}+i} a\left(y_{0}, \mathbf{m}\right) b\left(x_{0}, \mathbf{x}\right) c\left(z_{0}, \mathbf{z}\right)-\left(-x_{0}+y_{0}\right)^{m_{0}+i} b\left(x_{0}, \mathbf{x}\right) a\left(y_{0}, \mathbf{m}\right) c\left(z_{0}, \mathbf{z}\right)\right) \\
& \sum_{j=k+1}^{3 k}\left(\begin{array}{c}
3 k \\
j
\end{array}\right)\left(x_{0}-y_{0}\right)^{3 k-j}\left(y_{0}-z_{0}\right)^{j}\left(x_{0}-z_{0}\right)^{k} \cdot \\
= & \cdot\left(\left(y_{0}-x_{0}\right)^{m_{0}+i} a\left(y_{0}, \mathbf{m}\right) b\left(x_{0}, \mathbf{x}\right) c\left(z_{0}, \mathbf{z}\right)-\left(-x_{0}+y_{0}\right)^{m_{0}+i} b\left(x_{0}, \mathbf{x}\right) a\left(y_{0}, \mathbf{m}\right) c\left(z_{0}, \mathbf{z}\right)\right) \\
& \sum_{j=k+1}^{3 k}\left(\begin{array}{c}
3 k \\
j
\end{array}\right)\left(x_{0}-y_{0}\right)^{3 k-j}\left(y_{0}-z_{0}\right)^{j}\left(x_{0}-z_{0}\right)^{k} . \\
= & \quad\left(\left(y_{0}-x_{0}\right)^{m_{0}+i} c\left(z_{0}, \mathbf{z}\right) a\left(y_{0}, \mathbf{m}\right) b\left(x_{0}, \mathbf{x}\right)-\left(-x_{0}+y_{0}\right)^{m_{0}+i} c\left(z_{0}, \mathbf{z}\right) b\left(x_{0}, \mathbf{x}\right) a\left(y_{0}, \mathbf{m}\right)\right) \\
\quad & \left.\quad\left(-x_{0}+y_{0}\right)^{m_{0}+i} c\left(z_{0}, \mathbf{z}\right) b\left(x_{0}, \mathbf{x}\right) a\left(y_{0}, \mathbf{m}\right)\right),
\end{aligned}
$$


we get

$$
\left(x_{0}-z_{0}\right)^{4 k}\left(a\left(x_{0}, \mathbf{x}\right)_{m_{0}, \mathbf{m}} b\left(x_{0}, \mathbf{x}\right)\right) c\left(z_{0}, \mathbf{z}\right)=\left(x_{0}-z_{0}\right)^{4 k} c\left(z_{0}, \mathbf{z}\right)\left(a\left(x_{0}, \mathbf{x}\right)_{m_{0}, \mathbf{m}} b\left(x_{0}, \mathbf{x}\right)\right) .
$$

as desired.

A graded local subspace $U$ of $\mathcal{E}(W, r ; N)$ is said to be closed if

$$
1_{W} \in U \text { and } a\left(x_{0}, \mathbf{x}\right)_{m_{0}, \mathbf{m}} b\left(x_{0}, \mathbf{x}\right) \in U
$$

for all $a\left(x_{0}, \mathbf{x}\right), b\left(x_{0}, \mathbf{x}\right) \in U,\left(m_{0}, \mathbf{m}\right) \in \mathbb{Z} \times \mathbb{Z}^{r}$.

The following is the main result of this section:

Theorem 3.5. Let $V$ be a closed graded local subspace of $\mathcal{E}(W, r ; N)$. Then $\left(V, Y_{\mathcal{E}}, 1_{W}\right)$ carries the structure of an $(r+1)$-toroidal vertex algebra with $\sigma$ as an automorphism which has $N$ as a period and $W$ is a faithful $\sigma$-twisted $V$-module with

$$
Y_{W}\left(a\left(x_{0}, \mathbf{x}\right) ; z_{0}, \mathbf{z}\right)=a\left(z_{0}, \mathbf{z}\right) \quad \text { for } a\left(x_{0}, \mathbf{x}\right) \in V .
$$

Proof. For any $b\left(x_{0}, \mathbf{x}\right) \in \mathcal{E}(W, r ; N)$, from Definition 3.1 we have

$$
\begin{aligned}
& Y_{\mathcal{E}}\left(1_{W} ; z_{0}, \mathbf{z}\right) b\left(y_{0}, \mathbf{y}\right)=\operatorname{Res}_{x_{0}}\left(z_{0}^{-1} \delta\left(\frac{x_{0}-y_{0}}{z_{0}}\right)-z_{0}^{-1} \delta\left(\frac{y_{0}-x_{0}}{-z_{0}}\right)\right) b\left(y_{0}, \mathbf{y}\right) \\
& \quad=\operatorname{Res}_{x_{0}} x_{0}^{-1} \delta\left(\frac{y_{0}+z_{0}}{x_{0}}\right) b\left(y_{0}, \mathbf{y}\right)=b\left(y_{0}, \mathbf{y}\right) .
\end{aligned}
$$

On the other hand, for any $a\left(x_{0}, \mathbf{x}\right) \in \mathcal{E}(W, r ; N)_{s}$ with $0 \leq s<N$, we have

$$
\begin{aligned}
& Y_{\mathcal{E}}\left(a\left(y_{0}, \mathbf{y}\right) ; z_{0}, \mathbf{z}\right) 1_{W} \\
= & \operatorname{Res}_{x_{0}}\left(\frac{x_{0}-z_{0}}{y_{0}}\right)^{\frac{s}{N}}\left(z_{0}^{-1} \delta\left(\frac{x_{0}-y_{0}}{z_{0}}\right)-z_{0}^{-1} \delta\left(\frac{y_{0}-x_{0}}{-z_{0}}\right)\right) a\left(x_{0}, \mathbf{z y}\right) \\
= & \operatorname{Res}_{x_{0}} y_{0}^{-1} \delta\left(\frac{x_{0}-z_{0}}{y_{0}}\right)\left(\frac{x_{0}-z_{0}}{y_{0}}\right)^{\frac{s}{N}} a\left(x_{0}, \mathbf{z y}\right) \\
= & \operatorname{Res}_{x_{0}} x_{0}^{-1} \delta\left(\frac{y_{0}+z_{0}}{x_{0}}\right)\left(\frac{y_{0}+z_{0}}{x_{0}}\right)^{-\frac{s}{N}} a\left(x_{0}, \mathbf{z y}\right) \\
= & \left(y_{0}+z_{0}\right)^{-\frac{s}{N}} \operatorname{Res}_{x_{0}} x_{0}^{-1} \delta\left(\frac{y_{0}+z_{0}}{x_{0}}\right)\left(x_{0}^{\frac{s}{N}} a\left(x_{0}, \mathbf{z y}\right)\right. \\
= & a\left(y_{0}+z_{0}, \mathbf{z y}\right)=e^{z_{0} \frac{\partial}{\partial y_{0}}} a\left(y_{0}, \mathbf{z y}\right) .
\end{aligned}
$$

To prove the Jacobi identity, let $a\left(x_{0}, \mathbf{x}\right), b\left(x_{0}, \mathbf{x}\right), c\left(x_{0}, \mathbf{x}\right) \in V$ such that $a\left(x_{0}, \mathbf{x}\right) \in$ $\mathcal{E}\left(W, r ; N_{0}\right)_{\lambda}$ and $b\left(x_{0}, \mathbf{x}\right) \in \mathcal{E}\left(W, r ; N_{0}\right)_{\mu}$ with $0 \leq \lambda, \mu<N$. For simplicity, in the following, we write $\operatorname{Res}_{u_{0}, v_{0}}$ for $\operatorname{Res}_{u_{0}} \operatorname{Res}_{v_{0}}$. By Definition 3.1, we have

$$
\begin{aligned}
& z_{0}^{-1} \delta\left(\frac{x_{0}-y_{0}}{z_{0}}\right) Y_{\mathcal{E}}\left(a\left(t_{0}, \mathbf{t}\right) ; x_{0}, \mathbf{y z}\right) Y_{\mathcal{E}}\left(b\left(t_{0}, \mathbf{t}\right) ; y_{0}, \mathbf{y}\right) c\left(t_{0}, \mathbf{t}\right) \\
= & \operatorname{Res}_{u_{0}, v_{0}}\left(\frac{v_{0}-x_{0}}{t_{0}}\right)^{\frac{\lambda}{N}}\left(\frac{u_{0}-y_{0}}{t_{0}}\right)^{\frac{\mu}{N}} z_{0}^{-1} \delta\left(\frac{x_{0}-y_{0}}{z_{0}}\right) T
\end{aligned}
$$


where

$$
\begin{aligned}
T= & x_{0}^{-1} \delta\left(\frac{v_{0}-t_{0}}{x_{0}}\right) y_{0}^{-1} \delta\left(\frac{u_{0}-t_{0}}{y_{0}}\right) a\left(v_{0}, \mathbf{z y t}\right) b\left(u_{0}, \mathbf{y t}\right) c\left(t_{0}, \mathbf{t}\right) \\
& -x_{0}^{-1} \delta\left(\frac{v_{0}-t_{0}}{x_{0}}\right) y_{0}^{-1} \delta\left(\frac{t_{0}-u_{0}}{-y_{0}}\right) a\left(v_{0}, \mathbf{z y t}\right) c\left(t_{0}, \mathbf{t}\right) b\left(u_{0}, \mathbf{y t}\right) \\
& -x_{0}^{-1} \delta\left(\frac{t_{0}-v_{0}}{-x_{0}}\right) y_{0}^{-1} \delta\left(\frac{u_{0}-t_{0}}{y_{0}}\right) b\left(u_{0}, \mathbf{y t}\right) c\left(t_{0}, \mathbf{t}\right) a\left(v_{0}, \mathbf{z y t}\right) \\
& +x_{0}^{-1} \delta\left(\frac{t_{0}-v_{0}}{-x_{0}}\right) y_{0}^{-1} \delta\left(\frac{t_{0}-u_{0}}{-y_{0}}\right) c\left(t_{0}, \mathbf{t}\right) b\left(u_{0}, \mathbf{y t}\right) a\left(v_{0}, \mathbf{z y t}\right) .
\end{aligned}
$$

Let $k$ be a nonnegative integer such that

$$
\begin{aligned}
& \left(x_{0}-y_{0}\right)^{k}\left[a\left(x_{0}, \mathbf{x}\right), b\left(y_{0}, \mathbf{y}\right)\right]=0 \\
& \left(x_{0}-y_{0}\right)^{k}\left[a\left(x_{0}, \mathbf{x}\right), c\left(y_{0}, \mathbf{y}\right)\right]=0 \\
& \left(x_{0}-y_{0}\right)^{k}\left[b\left(x_{0}, \mathbf{x}\right), c\left(y_{0}, \mathbf{y}\right)\right]=0
\end{aligned}
$$

Using the basic delta-function substitution property we get

$$
\begin{aligned}
& x_{0}^{k} y_{0}^{k} z_{0}^{k} z_{0}^{-1} \delta\left(\frac{x_{0}-y_{0}}{z_{0}}\right) T=z_{0}^{-1} \delta\left(\frac{x_{0}-y_{0}}{z_{0}}\right)\left(v_{0}-t_{0}\right)^{k}\left(u_{0}-t_{0}\right)^{k}\left(v_{0}-u_{0}\right)^{k} T \\
= & z_{0}^{-1} \delta\left(\frac{x_{0}-y_{0}}{z_{0}}\right) t_{0}^{-1} \delta\left(\frac{v_{0}-x_{0}}{t_{0}}\right) t_{0}^{-1} \delta\left(\frac{u_{0}-y_{0}}{t_{0}}\right) \\
& \cdot\left(v_{0}-t_{0}\right)^{k}\left(u_{0}-t_{0}\right)^{k}\left(v_{0}-u_{0}\right)^{k} a\left(v_{0}, \mathbf{z y t}\right) b\left(u_{0}, \mathbf{y t}\right) c\left(t_{0}, \mathbf{t}\right) .
\end{aligned}
$$

Consequently,

$$
\begin{aligned}
& x_{0}^{k} y_{0}^{k} z_{0}^{k} z_{0}^{-1} \delta\left(\frac{x_{0}-y_{0}}{z_{0}}\right) Y_{\mathcal{E}}\left(a\left(t_{0}, \mathbf{t}\right) ; x_{0}, \mathbf{y z}\right) Y_{\mathcal{E}}\left(b\left(t_{0}, \mathbf{t}\right) ; y_{0}, \mathbf{y}\right) c\left(t_{0}, \mathbf{t}\right) \\
= & \operatorname{Res}_{u_{0}, v_{0}} z_{0}^{-1} \delta\left(\frac{x_{0}-y_{0}}{z_{0}}\right) t_{0}^{-1} \delta\left(\frac{v_{0}-x_{0}}{t_{0}}\right)\left(\frac{v_{0}-x_{0}}{t_{0}}\right)^{\frac{\lambda}{N}} t_{0}^{-1} \delta\left(\frac{u_{0}-y_{0}}{t_{0}}\right)\left(\frac{u_{0}-y_{0}}{t_{0}}\right)^{\frac{\mu}{N}} S,
\end{aligned}
$$

where

$$
S=\left(v_{0}-t_{0}\right)^{k}\left(u_{0}-t_{0}\right)^{k}\left(v_{0}-u_{0}\right)^{k} a\left(v_{0}, \mathbf{z y t}\right) b\left(u_{0}, \mathbf{y t}\right) c\left(t_{0}, \mathbf{t}\right) .
$$

Similarly, we have

$$
\begin{aligned}
& x_{0}^{k} y_{0}^{k} z_{0}^{k} z_{0}^{-1} \delta\left(\frac{y_{0}-x_{0}}{-z_{0}}\right) Y_{\mathcal{E}}\left(b\left(t_{0}, \mathbf{t}\right) ; y_{0}, \mathbf{y}\right) Y_{\mathcal{E}}\left(a\left(t_{0}, \mathbf{t}\right) ; x_{0}, \mathbf{y z}\right) c\left(t_{0}, \mathbf{t}\right) \\
= & \operatorname{Res}_{u_{0}, v_{0}} z_{0}^{-1} \delta\left(\frac{y_{0}-x_{0}}{-z_{0}}\right) t_{0}^{-1} \delta\left(\frac{v_{0}-x_{0}}{t_{0}}\right)\left(\frac{v_{0}-x_{0}}{t_{0}}\right)^{\frac{\lambda}{N}} t_{0}^{-1} \delta\left(\frac{u_{0}-y_{0}}{t_{0}}\right)\left(\frac{u_{0}-y_{0}}{t_{0}}\right)^{\frac{\mu}{N}} S .
\end{aligned}
$$


On the other hand, we have

$$
\begin{aligned}
& y_{0}^{-1} \delta\left(\frac{x_{0}-z_{0}}{y_{0}}\right) Y_{\mathcal{E}}\left(Y_{\mathcal{E}}\left(a\left(t_{0}, \mathbf{t}\right) ; z_{0}, \mathbf{z}\right) b\left(t_{0}, \mathbf{t}\right) ; y_{0}, \mathbf{y}\right) c\left(t_{0}, \mathbf{t}\right) \\
= & \operatorname{Res}_{u_{0}, v_{0}}\left(\frac{v_{0}-z_{0}}{u_{0}}\right)^{\frac{\lambda}{N}}\left(\frac{u_{0}-y_{0}}{t_{0}}\right)^{\frac{\lambda+\mu}{N}} y_{0}^{-1} \delta\left(\frac{x_{0}-z_{0}}{y_{0}}\right) T^{\prime},
\end{aligned}
$$

where

$$
\begin{aligned}
T^{\prime}= & z_{0}^{-1} \delta\left(\frac{v_{0}-u_{0}}{z_{0}}\right) y_{0}^{-1} \delta\left(\frac{u_{0}-t_{0}}{y_{0}}\right) a\left(v_{0}, \mathbf{z y t}\right) b\left(u_{0}, \mathbf{y t}\right) c\left(t_{0}, \mathbf{t}\right) \\
& -z_{0}^{-1} \delta\left(\frac{u_{0}-v_{0}}{-z_{0}}\right) y_{0}^{-1} \delta\left(\frac{u_{0}-t_{0}}{y_{0}}\right) b\left(u_{0}, \mathbf{y t}\right) a\left(v_{0}, \mathbf{z y t}\right) c\left(t_{0}, \mathbf{t}\right) \\
& -z_{0}^{-1} \delta\left(\frac{v_{0}-u_{0}}{z_{0}}\right) y_{0}^{-1} \delta\left(\frac{t_{0}-u_{0}}{y_{0}}\right) c\left(t_{0}, \mathbf{t}\right) a\left(v_{0}, \mathbf{z y t}\right) b\left(u_{0}, \mathbf{y t}\right) \\
& +z_{0}^{-1} \delta\left(\frac{u_{0}-v_{0}}{-z_{0}}\right) y_{0}^{-1} \delta\left(\frac{t_{0}-u_{0}}{y_{0}}\right) c\left(t_{0}, \mathbf{t}\right) b\left(u_{0}, \mathbf{y t}\right) a\left(v_{0}, \mathbf{z y t}\right),
\end{aligned}
$$

and furthermore we have

$$
\begin{aligned}
& x_{0}^{k} y_{0}^{k} z_{0}^{k} y_{0}^{-1} \delta\left(\frac{x_{0}-z_{0}}{y_{0}}\right) Y_{\mathcal{E}}\left(Y_{\mathcal{E}}\left(a\left(t_{0}, \mathbf{t}\right) ; z_{0}, \mathbf{z}\right) b\left(t_{0}, \mathbf{t}\right) ; y_{0}, \mathbf{y}\right) c\left(t_{0}, \mathbf{t}\right) \\
= & \operatorname{Res}_{u_{0}, v_{0}} y_{0}^{-1} \delta\left(\frac{x_{0}-z_{0}}{y_{0}}\right) u_{0}^{-1} \delta\left(\frac{v_{0}-z_{0}}{u_{0}}\right)\left(\frac{v_{0}-z_{0}}{u_{0}}\right)^{\frac{\lambda}{N}} t_{0}^{-1} \delta\left(\frac{u_{0}-y_{0}}{t_{0}}\right)\left(\frac{u_{0}-y_{0}}{t_{0}}\right)^{\frac{\lambda+\mu}{N}} S .
\end{aligned}
$$


Note that for any $\alpha \in \mathbb{C},(x+y)^{\alpha}$ involves only integer powers of $y$. Then we have

$$
\begin{aligned}
& x_{0}^{k} y_{0}^{k} z_{0}^{k} z_{0}^{-1} \delta\left(\frac{x_{0}-y_{0}}{z_{0}}\right) Y_{\mathcal{E}}\left(a\left(t_{0}, \mathbf{t}\right) ; x_{0}, \mathbf{y z}\right) Y_{\mathcal{E}}\left(b\left(t_{0}, \mathbf{t}\right) ; y_{0}, \mathbf{y}\right) c\left(t_{0}, \mathbf{t}\right) \\
& -x_{0}^{k} y_{0}^{k} z_{0}^{k} z_{0}^{-1} \delta\left(\frac{y_{0}-x_{0}}{-z_{0}}\right) Y_{\mathcal{E}}\left(b\left(t_{0}, \mathbf{t}\right) ; y_{0}, \mathbf{y}\right) Y_{\mathcal{E}}\left(a\left(t_{0}, \mathbf{t}\right) ; x_{0}, \mathbf{y z}\right) c\left(t_{0}, \mathbf{t}\right) \\
& =\operatorname{Res}_{u_{0}, v_{0}} y_{0}^{-1} \delta\left(\frac{x_{0}-z_{0}}{y_{0}}\right) t_{0}^{-1} \delta\left(\frac{v_{0}-x_{0}}{t_{0}}\right)\left(\frac{v_{0}-x_{0}}{t_{0}}\right)^{\frac{\lambda}{N}} t_{0}^{-1} \delta\left(\frac{u_{0}-y_{0}}{t_{0}}\right)\left(\frac{u_{0}-y_{0}}{t_{0}}\right)^{\frac{\mu}{N}} S \\
& =\operatorname{Res}_{u_{0}, v_{0}} x_{0}^{-1} \delta\left(\frac{y_{0}+z_{0}}{x_{0}}\right) v_{0}^{-1} \delta\left(\frac{t_{0}+x_{0}}{v_{0}}\right)\left(\frac{t_{0}+x_{0}}{v_{0}}\right)^{-\frac{\lambda}{N}} u_{0}^{-1} \delta\left(\frac{t_{0}+y_{0}}{u_{0}}\right)\left(\frac{t_{0}+y_{0}}{u_{0}}\right)^{-\frac{\mu}{N}} S \\
& =\operatorname{Res}_{u_{0}, v_{0}} x_{0}^{-1} \delta\left(\frac{y_{0}+z_{0}}{x_{0}}\right) v_{0}^{-1} \delta\left(\frac{t_{0}+y_{0}+z_{0}}{v_{0}}\right)\left(\frac{t_{0}+y_{0}+z_{0}}{v_{0}}\right)^{-\frac{\lambda}{N}}\left(\frac{t_{0}+y_{0}}{u_{0}}\right)^{\frac{\lambda}{N}} \\
& \cdot u_{0}^{-1} \delta\left(\frac{t_{0}+y_{0}}{u_{0}}\right)\left(\frac{t_{0}+y_{0}}{u_{0}}\right)^{-\frac{\lambda+\mu}{N}} S \\
& =\operatorname{Res}_{u_{0}, v_{0}} x_{0}^{-1} \delta\left(\frac{y_{0}+z_{0}}{x_{0}}\right) v_{0}^{-1} \delta\left(\frac{t_{0}+y_{0}+z_{0}}{v_{0}}\right)\left(\frac{1+z_{0}\left(t_{0}+y_{0}\right)^{-1}}{v_{0}}\right)^{-\frac{\lambda}{N}} u_{0}^{-\frac{\lambda}{N}} \\
& \cdot u_{0}^{-1} \delta\left(\frac{t_{0}+y_{0}}{u_{0}}\right)\left(\frac{t_{0}+y_{0}}{u_{0}}\right)^{-\frac{\lambda+\mu}{N}} S \\
& =\operatorname{Res}_{u_{0}, v_{0}} x_{0}^{-1} \delta\left(\frac{y_{0}+z_{0}}{x_{0}}\right) v_{0}^{-1} \delta\left(\frac{u_{0}+z_{0}}{v_{0}}\right)\left(\frac{u_{0}+z_{0}}{v_{0}}\right)^{-\frac{\lambda}{N}} u_{0}^{-1} \delta\left(\frac{t_{0}+y_{0}}{u_{0}}\right)\left(\frac{t_{0}+y_{0}}{u_{0}}\right)^{-\frac{\lambda+\mu}{N}} S \\
& =\operatorname{Res}_{u_{0}, v_{0}} y_{0}^{-1} \delta\left(\frac{x_{0}-z_{0}}{y_{0}}\right) u_{0}^{-1} \delta\left(\frac{v_{0}-z_{0}}{u_{0}}\right)\left(\frac{v_{0}-z_{0}}{u_{0}}\right)^{\frac{\lambda}{N}} t_{0}^{-1} \delta\left(\frac{u_{0}-y_{0}}{t_{0}}\right)\left(\frac{u_{0}-y_{0}}{t_{0}}\right)^{\frac{\lambda+\mu}{N}} S \\
& =x_{0}^{k} y_{0}^{k} z_{0}^{k} y_{0}^{-1} \delta\left(\frac{x_{0}-z_{0}}{y_{0}}\right) Y_{\mathcal{E}}\left(Y_{\mathcal{E}}\left(a\left(t_{0}, \mathbf{t}\right) ; z_{0}, \mathbf{z}\right) b\left(t_{0}, \mathbf{t}\right) ; y_{0}, \mathbf{y}\right) c\left(t_{0}, \mathbf{t}\right) \text {. }
\end{aligned}
$$

Thus

$$
\begin{aligned}
z_{0}^{-1} \delta & \left(\frac{x_{0}-y_{0}}{z_{0}}\right) Y_{\mathcal{E}}\left(a\left(t_{0}, \mathbf{t}\right) ; x_{0}, \mathbf{y z}\right) Y_{\mathcal{E}}\left(b\left(t_{0}, \mathbf{t}\right) ; y_{0}, \mathbf{y}\right) c\left(t_{0}, \mathbf{t}\right) \\
& -z_{0}^{-1} \delta\left(\frac{y_{0}-x_{0}}{-z_{0}}\right) Y_{\mathcal{E}}\left(b\left(t_{0}, \mathbf{t}\right) ; y_{0}, \mathbf{y}\right) Y_{\mathcal{E}}\left(a\left(t_{0}, \mathbf{t}\right) ; x_{0}, \mathbf{y z}\right) c\left(t_{0}, \mathbf{t}\right) \\
= & x_{0}^{-1} \delta\left(\frac{y_{0}+z_{0}}{x_{0}}\right) Y_{\mathcal{E}}\left(Y_{\mathcal{E}}\left(a\left(t_{0}, \mathbf{t}\right) ; z_{0}, \mathbf{z}\right) b\left(t_{0}, \mathbf{t}\right) ; y_{0}, \mathbf{y}\right) c\left(t_{0}, \mathbf{t}\right) .
\end{aligned}
$$

This proves that the Jacobi identity holds. Therefore, $\left(V, Y_{\mathcal{E}}, 1_{W}\right)$ carries the structure of an $(r+1)$-toroidal vertex algebra.

As $V$ is a graded subspace of $\mathcal{E}(W, r ; N), \sigma$ is naturally a linear automorphism of $V$. It follows from Lemma 3.2 that $\sigma$ is an automorphism of $V$ viewed as an $(r+1)$-toroidal vertex algebra and $\sigma^{N}=1$. 
For $a\left(x_{0}, \mathbf{x}\right) \in V$, we set

$$
Y_{W}\left(a\left(x_{0}, \mathbf{x}\right) ; z_{0}, \mathbf{z}\right)=a\left(z_{0}, \mathbf{z}\right) .
$$

For $a\left(x_{0}, \mathbf{x}\right), b\left(x_{0}, \mathbf{x}\right) \in V$, from assumption they are mutually local. To show that $\left(W, Y_{W}\right)$ is a $\sigma$-twisted $V$-module, in view of Lemma 2.5 we need to establish weak associativity. Let $k$ be a nonnegative integer such that

$$
\left(x_{0}-y_{0}\right)^{k} a\left(x_{0}, \mathbf{x}\right) b\left(y_{0}, \mathbf{y}\right)=\left(x_{0}-y_{0}\right)^{k} b\left(y_{0}, \mathbf{y}\right) a\left(x_{0}, \mathbf{x}\right) .
$$

Assume $a\left(x_{0}, \mathbf{x}\right) \in \mathcal{E}(W, r ; N)_{s}$ with $0 \leq s<N$. Let $w \in W$ be an arbitrarily fixed vector in $W$ and let $\ell$ be a nonnegative integer such that

$$
x_{0}^{\ell+\frac{s}{N}} a\left(x_{0}, \mathbf{x}\right) w \in W\left[\left[x_{0}, \mathbf{x}, \mathbf{x}^{-1}\right]\right] .
$$

From Remark 3.3, we have

$$
\begin{aligned}
& z_{0}^{k}\left(y_{0}+z_{0}\right)^{\ell+\frac{s}{N}}\left(Y_{\mathcal{E}}\left(a\left(y_{0}, \mathbf{y}\right) ; z_{0}, \mathbf{z}\right) b\left(y_{0}, \mathbf{y}\right)\right) w \\
= & {\left.\left[\left(x_{0}-y_{0}\right)^{k} x_{0}^{\ell+\frac{s}{N}} a\left(x_{0}, \mathbf{z y}\right) b\left(y_{0}, \mathbf{y}\right) w\right]\right|_{x_{0}=y_{0}+z_{0}} } \\
= & {\left.\left[\left(x_{0}-y_{0}\right)^{k} x_{0}^{\ell+\frac{s}{N}} a\left(x_{0}, \mathbf{z y}\right) b\left(y_{0}, \mathbf{y}\right) w\right]\right|_{x_{0}=z_{0}+y_{0}} } \\
= & z_{0}^{k}\left(z_{0}+y_{0}\right)^{\ell+\frac{s}{N}} a\left(z_{0}+y_{0}, \mathbf{y z}\right) b\left(y_{0}, \mathbf{y}\right) w .
\end{aligned}
$$

This proves

$$
\begin{aligned}
& \left(y_{0}+z_{0}\right)^{\ell+\frac{s}{N}} Y_{W}\left(Y_{\mathcal{E}}\left(a\left(t_{0}, \mathbf{t}\right) ; z_{0}, \mathbf{z}\right) b\left(t_{0}, \mathbf{t}\right) ; y_{0}, \mathbf{y}\right) \\
= & \left(y_{0}+z_{0}\right)^{\ell+\frac{s}{N}} Y_{\mathcal{E}}\left(a\left(y_{0}, \mathbf{y}\right) ; z_{0}, \mathbf{z}\right) b\left(y_{0}, \mathbf{y}\right) \\
= & \left(z_{0}+y_{0}\right)^{\ell+\frac{s}{N}} a\left(z_{0}+y_{0}, \mathbf{y z}\right) b\left(y_{0}, \mathbf{y}\right) \\
= & \left(z_{0}+y_{0}\right)^{\ell+\frac{s}{N}} Y_{W}\left(a\left(t_{0}, \mathbf{t}\right) ; z_{0}+y_{0}, \mathbf{y z}\right) Y_{W}\left(b\left(t_{0}, \mathbf{t}\right) ; y_{0}, \mathbf{y}\right) .
\end{aligned}
$$

By Lemma 2.5, $W$ is a $\sigma$-twisted $V$-module. It is clear that $W$ is faithful.

Using Lemma 3.4 and Theorem 3.5, we immediately have (cf. [Li2]):

Corollary 3.6. Let $U$ be any graded local subspace of $\mathcal{E}(W, r ; N)$. Then there exists a smallest closed graded local subspace $\langle U\rangle$ that contains $U$, and $\langle U\rangle$ is an $(r+1)$ toroidal vertex algebra with $W$ as a canonical faithful $\sigma$-twisted module.

In view of Theorem 3.5, we alternatively call a closed graded local subspace of $\mathcal{E}(W, r ; N)$ an $(r+1)$-toroidal vertex subalgebra of $\mathcal{E}(W, r ; N)$.

Let $V$ be any $(r+1)$-toroidal vertex subalgebra of $\mathcal{E}(W, r ; N)$. Note that by Theorem 3.5. $\left(W, Y_{W}\right)$ is a faithful $\sigma$-twisted $V$-module with $Y_{W}\left(a\left(x_{0}, \mathbf{x}\right) ; z_{0}, \mathbf{z}\right)=$ $a\left(z_{0}, \mathbf{z}\right)$ for $a\left(x_{0}, \mathbf{x}\right) \in V$. In view of Lemma 2.6, we immediately have: 
Corollary 3.7. Let $V$ be an $(r+1)$-toroidal vertex subalgebra of $\mathcal{E}(W, r ; N)$, let

$$
a\left(x_{0}, \mathbf{x}\right), b\left(x_{0}, \mathbf{x}\right), c_{0}\left(x_{0}, \mathbf{x}\right), c_{1}\left(x_{0}, \mathbf{x}\right), \ldots, c_{k}\left(x_{0}, \mathbf{x}\right) \in V
$$

and let $\mathbf{m} \in \mathbb{Z}^{r}$. Assume $a\left(x_{0}, \mathbf{x}\right) \in \mathcal{E}(W, r ; N)_{s}$ with $0 \leq s<N$. If

$$
\left[a\left(x_{0}, \mathbf{m}\right), b\left(y_{0}, \mathbf{y}\right)\right]=\mathbf{y}^{\mathbf{m}} \sum_{j=0}^{k} c_{j}\left(y_{0}, \mathbf{y}\right) \frac{1}{j !}\left(\frac{\partial}{\partial y_{0}}\right)^{j}\left(x_{0}^{-1} \delta\left(\frac{y_{0}}{x_{0}}\right)\left(\frac{y_{0}}{x_{0}}\right)^{\frac{s}{N}}\right),
$$

where $a\left(x_{0}, \mathbf{x}\right)=\sum_{\mathbf{n} \in \mathbb{Z}^{r}} a\left(x_{0}, \mathbf{n}\right) \mathbf{x}^{-\mathbf{n}}$, then

$$
a\left(x_{0}, \mathbf{x}\right)_{j, \mathbf{m}} b\left(x_{0}, \mathbf{x}\right)=c_{j}\left(x_{0}, \mathbf{x}\right)
$$

for $0 \leq j \leq k$ and $a\left(x_{0}, \mathbf{x}\right)_{j, \mathbf{m}} b\left(x_{0}, \mathbf{x}\right)=0$ for $j>k$. Furthermore, we have

$$
\begin{aligned}
& {\left[Y_{\mathcal{E}}\left(a\left(t_{0}, \mathbf{t}\right) ; x_{0}, \mathbf{m}\right), Y_{\mathcal{E}}\left(b\left(t_{0}, \mathbf{t}\right) ; y_{0}, \mathbf{y}\right)\right] } \\
= & \mathbf{y}^{\mathbf{m}} \sum_{j=0}^{k} Y_{\mathcal{E}}\left(c_{j}\left(t_{0}, \mathbf{t}\right) ; y_{0}, \mathbf{y}\right) \frac{1}{j !}\left(\frac{\partial}{\partial y_{0}}\right)^{j} x_{0}^{-1} \delta\left(\frac{y_{0}}{x_{0}}\right) .
\end{aligned}
$$

\section{4 ( $r+1)$-toroidal vertex algebras and twisted mod- ules associated to twisted toroidal Lie algebras}

In this section, we shall associate $(r+1)$-toroidal vertex algebras and their twisted modules to certain twisted toroidal Lie algebras by using the general construction established in Section 3 ,

Let $\mathfrak{g}$ be a finite dimensional simple Lie algebra and let $\langle\cdot, \cdot\rangle$ be the killing form which is normalized such that $\langle\alpha, \alpha\rangle=2$ for any long root $\alpha$. Let $\sigma_{0}, \sigma_{1}, \ldots, \sigma_{r}$ be $r+1$ mutually commuting finite order automorphisms of $\mathfrak{g}$, which are fixed throughout this section.

Set $o\left(\sigma_{i}\right)=N_{i}$ for $0 \leq i \leq r$ and set

$$
\begin{aligned}
G & =\left\langle\sigma_{0}, \sigma_{1}, \ldots, \sigma_{r}\right\rangle \subset \operatorname{Aut}(\mathfrak{g}), \\
G_{+} & =\left\langle\sigma_{1}, \sigma_{2}, \ldots, \sigma_{r}\right\rangle \subset G .
\end{aligned}
$$

Note that every automorphism of $\mathfrak{g}$ preserves the killing form (cf. [K]) and symmetric invariant bilinear forms on $\mathfrak{g}$ are unique up to scalar multiples (as $\mathfrak{g}$ is simple). It follows that all $\sigma_{i}$ for $0 \leq i \leq r$ preserve $\langle\cdot, \cdot\rangle$.

Consider the following $(r+1)$-loop Lie algebra

$$
L_{r+1}\left(\mathfrak{g}, N_{0}\right)=\mathfrak{g} \otimes \mathbb{C}\left[t_{0}^{ \pm \frac{1}{N_{0}}}, t_{1}^{ \pm 1}, \ldots, t_{r}^{ \pm 1}\right] .
$$

Form a 1-dimensional central extension

$$
\widehat{L_{r+1}}\left(\mathfrak{g}, N_{0}\right)=L_{r+1}\left(\mathfrak{g}, N_{0}\right) \oplus \mathbb{C} \mathfrak{c},
$$


where $\mathfrak{c}$ is central and

$$
\left[a \otimes t_{0}^{m_{0}^{\prime}} \mathbf{t}^{\mathbf{m}}, b \otimes t_{0}^{n_{0}^{\prime}} \mathbf{t}^{\mathbf{n}}\right]=[a, b] \otimes t_{0}^{m_{0}^{\prime}+n_{0}^{\prime}} \mathbf{t}^{\mathbf{m}+\mathbf{n}}+m_{0}^{\prime}\langle a, b\rangle \delta_{m_{0}^{\prime}+n_{0}^{\prime}, 0} \delta_{\mathbf{m}+\mathbf{n}, 0} \mathfrak{c}
$$

for $a, b \in \mathfrak{g}, m_{0}^{\prime}, n_{0}^{\prime} \in \frac{1}{N_{0}} \mathbb{Z}, \mathbf{m}, \mathbf{n} \in \mathbb{Z}^{r}$.

Definition 4.1. For $0 \leq i \leq r$, we define an automorphism $\hat{\sigma}_{i}$ of the Lie algebra $\widehat{L_{r+1}}\left(\mathfrak{g}, N_{0}\right)$ by

$$
\hat{\sigma}_{i}(\mathfrak{c})=\mathfrak{c}, \quad \hat{\sigma}_{i}\left(a \otimes t_{0}^{\frac{m_{0}}{N_{0}}} \mathbf{t}^{\mathbf{m}}\right)=\omega_{N_{i}}^{-m_{i}}\left(\sigma_{i}(a) \otimes t_{0}^{\frac{m_{0}}{N_{0}}} \mathbf{t}^{\mathbf{m}}\right)
$$

for $a \in \mathfrak{g},\left(m_{0}, \mathbf{m}\right) \in \mathbb{Z} \times \mathbb{Z}^{r}$ with $\mathbf{m}=\left(m_{1}, \ldots, m_{r}\right)$.

Note that $\hat{\sigma}_{i}$ depends on both the index $i$ and the corresponding automorphism $\sigma_{i}$. We particularly mention that for $0 \leq i \neq j \leq r, \hat{\sigma}_{i}$ and $\hat{\sigma}_{j}$ are different even though $\sigma_{i}$ and $\sigma_{j}$ could be the same. The notation $\hat{\sigma}_{i}$, which is somewhat misleading, is for the purpose of convenience.

Set

$$
\begin{aligned}
& \widehat{G}=\left\langle\hat{\sigma}_{0}, \hat{\sigma}_{1}, \ldots, \hat{\sigma}_{r}\right\rangle \subset \operatorname{Aut}\left(\widehat{L_{r+1}}\left(\mathfrak{g}, N_{0}\right)\right), \\
& \widehat{G}_{+}=\left\langle\hat{\sigma}_{1}, \ldots, \hat{\sigma}_{r}\right\rangle \subset \widehat{G} .
\end{aligned}
$$

It is straightforawrd to show that

$$
\widehat{G} \simeq\left\langle\hat{\sigma}_{0}\right\rangle \times\left\langle\hat{\sigma}_{1}\right\rangle \times \cdots \times\left\langle\hat{\sigma}_{r}\right\rangle \text { and } \widehat{G}_{+} \simeq\left\langle\hat{\sigma}_{1}\right\rangle \times\left\langle\hat{\sigma}_{2}\right\rangle \times \cdots \times\left\langle\hat{\sigma}_{r}\right\rangle .
$$

Set $\mathbf{N}=\left(N_{1}, \ldots, N_{r}\right)$. Furthermore, we set

$$
\Lambda(\mathbf{N})=\mathbb{Z} N_{1} \times \cdots \times \mathbb{Z} N_{r} \subset \mathbb{Z}^{r}
$$

Then

$$
\mathbb{Z}^{r} / \Lambda(\mathbf{N}) \simeq \mathbb{Z}_{N_{1}} \times \cdots \times \mathbb{Z}_{N_{r}} \simeq\left\langle\hat{\sigma}_{1}\right\rangle \times\left\langle\hat{\sigma}_{2}\right\rangle \times \cdots \times\left\langle\hat{\sigma}_{r}\right\rangle=\widehat{G}_{+}
$$

Note that $G$ is a homomorphism image of $\widehat{G}$ with $\hat{\sigma}_{i}$ corresponding to $\sigma_{i}$ for $0 \leq i \leq r$. Then $\widehat{G}$ (and $\widehat{G}_{+}$) naturally acts on $\mathfrak{g}$ by automorphisms.

Set

$$
\tau=\left(\widehat{L_{r+1}}\left(\mathfrak{g}, N_{0}\right)\right)^{\widehat{G}}
$$

the subalgebra of $\widehat{G}$-fixed points in $\widehat{L_{r+1}}\left(\mathfrak{g}, N_{0}\right)$. Then

$$
\tau=\left(\bigoplus_{\left(m_{0}, \mathbf{m}\right) \in \mathbb{Z} \times \mathbb{Z}^{r}} \mathfrak{g}_{m_{0}, \mathbf{m}} \otimes t_{0}^{\frac{m_{0}}{N_{0}}} \mathbf{t}^{\mathbf{m}}\right) \oplus \mathbb{C} \mathfrak{c}
$$


where for $m_{0} \in \mathbb{Z}, \mathbf{m}=\left(m_{1}, \ldots, m_{r}\right) \in \mathbb{Z}^{r}$,

$$
\mathfrak{g}_{m_{0}, \mathbf{m}}=\left\{a \in \mathfrak{g} \mid \sigma_{i}(a)=\omega_{N_{i}}^{m_{i}} a \text { for } 0 \leq i \leq r\right\} .
$$

For $a \in \mathfrak{g}_{k_{0}, \mathbf{k}}$ with $\left(k_{0}, \mathbf{k}\right) \in \mathbb{Z} \times \mathbb{Z}^{r}$, set

$$
a^{\tau}\left(x_{0}, \mathbf{x}\right)=\sum_{m_{0} \in \mathbb{Z},}\left(a \otimes t_{0}^{\frac{k_{0}}{N_{0}}+m_{0}} \mathbf{t}^{\mathbf{m}}\right) x_{0}^{-\frac{k_{0}}{N_{0}}-m_{0}-1} \mathbf{x}^{-\mathbf{m}}
$$

and we also set

$$
a^{\tau}\left(x_{0}, \mathbf{m}\right)=\sum_{m_{0} \in \mathbb{Z}}\left(a \otimes t_{0}^{\frac{k_{0}}{N_{0}}+m_{0}} \mathbf{t}^{\mathbf{m}}\right) x_{0}^{-\frac{k_{0}}{N_{0}}-m_{0}-1}
$$

for $\mathbf{m} \in \mathbf{k}+\Lambda(\mathbf{N})$, and define $a^{\tau}\left(x_{0}, \mathbf{m}\right)=0$ for $\mathbf{m} \notin \mathbf{k}+\Lambda(\mathbf{N})$. We then define $a^{\tau}\left(x_{0}, \mathbf{x}\right)$ for general $a \in \mathfrak{g}$ by linearity.

For $a \in \mathfrak{g}_{k_{0}, \mathbf{k}}, b \in \mathfrak{g}_{\ell_{0}, \mathbf{l}}, \mathbf{m} \in \mathbf{k}+\Lambda(\mathbf{N}) \subset \mathbb{Z}^{r}$, we have

$$
\begin{aligned}
{\left[a^{\tau}\left(x_{0}, \mathbf{m}\right), b^{\tau}\left(y_{0}, \mathbf{y}\right)\right]=} & \mathbf{y}^{\mathbf{m}}[a, b]^{\tau}\left(y_{0}, \mathbf{y}\right) x_{0}^{-1} \delta\left(\frac{y_{0}}{x_{0}}\right)\left(\frac{y_{0}}{x_{0}}\right)^{\frac{k_{0}}{N_{0}}} \\
& +\mathbf{y}^{\mathbf{m}}\langle a, b\rangle \mathfrak{c} \frac{\partial}{\partial y_{0}}\left(x_{0}^{-1} \delta\left(\frac{y_{0}}{x_{0}}\right)\left(\frac{y_{0}}{x_{0}}\right)^{\frac{k_{0}}{N_{0}}}\right) .
\end{aligned}
$$

Note that for $\mathbf{m} \notin \mathbf{k}+\Lambda(\mathbf{N})$, we have $\left[a^{\tau}\left(x_{0}, \mathbf{m}\right), b^{\tau}\left(y_{0}, \mathbf{y}\right)\right]=0$.

Let $\ell \in \mathbb{C}$. A $\tau$-module $W$ is said to be of level $\ell$ if $\mathfrak{c}$ acts on $W$ as scalar $\ell$, and $W$ is called a restricted module if

$$
a^{\tau}\left(x_{0}, \mathbf{x}\right) \in \mathcal{E}\left(W, r ; N_{0}\right) \quad \text { for all } a \in \mathfrak{g} .
$$

Let $W$ be a restricted $\tau$-module of level $\ell$. Set

$$
U_{W}=\operatorname{Span}\left\{a^{\tau}\left(x_{0}, \mathbf{x}\right) \mid a \in \mathfrak{g}\right\}
$$

It follows from (4.15) that $U_{W}$ is a graded local subspace of $\mathcal{E}\left(W, r ; N_{0}\right)$. In view of Corollary 3.6, $U_{W}$ generates an $(r+1)$-toroidal vertex subalgebra $\left\langle U_{W}\right\rangle$ of $\mathcal{E}\left(W, r ; N_{0}\right)$ and $W$ is a faithful $\sigma$-twisted $\left\langle U_{W}\right\rangle$-module with $Y_{W}\left(\alpha\left(x_{0}, \mathbf{x}\right) ; z_{0}, \mathbf{z}\right)=\alpha\left(z_{0}, \mathbf{z}\right)$ for $\alpha\left(x_{0}, \mathbf{x}\right) \in\left\langle U_{W}\right\rangle$. In the following, we are going to characterize $\left\langle U_{W}\right\rangle$ as a module for another Lie algebra.

Consider the $(r+1)$-loop algebra

$$
L_{r+1}(\mathfrak{g})=\mathfrak{g} \otimes \mathbb{C}\left[t_{0}^{ \pm 1}, t_{1}^{ \pm 1}, \ldots, t_{r}^{ \pm 1}\right]
$$

which is equal to $L_{r+1}\left(\mathfrak{g}, N_{0}\right)$ with $N_{0}=1$. As before, $\widehat{G}_{+}$acts on $\widehat{L_{r+1}}(\mathfrak{g})$ by automorphisms. Set

$$
\mathcal{L}=\left(\widehat{L_{r+1}}(\mathfrak{g})\right)^{\widehat{G}_{+}}
$$


the subalgebra of $\widehat{G}_{+}$-fixed points in $\widehat{L_{r+1}}(\mathfrak{g})$. Then

$$
\mathcal{L}=\left(\bigoplus_{\mathbf{m} \in \mathbb{Z}^{r}} \mathfrak{g}_{\mathbf{m}} \otimes \mathbf{t}^{\mathbf{m}} \mathbb{C}\left[t_{0}^{ \pm 1}\right]\right) \bigoplus \mathbb{C} \mathfrak{c}
$$

where for $\mathbf{m}=\left(m_{1}, \ldots, m_{r}\right) \in \mathbb{Z}^{r}$,

$$
\mathfrak{g}_{\mathbf{m}}=\left\{a \in \mathfrak{g} \mid \sigma_{j}(a)=\omega_{N_{j}}^{m_{j}} a \text { for } 1 \leq j \leq r\right\} .
$$

Set

$$
\begin{aligned}
\mathcal{L}^{\geq 0} & =\left(\bigoplus_{\mathbf{m} \in \mathbb{Z}^{r}} \mathfrak{g}_{\mathbf{m}} \otimes \mathbf{t}^{\mathbf{m}} \mathbb{C}\left[t_{0}\right]\right) \oplus \mathbb{C} \mathfrak{c}, \\
\mathcal{L}^{-} & =\bigoplus_{\mathbf{m} \in \mathbb{Z}^{r}} \mathfrak{g}_{\mathbf{m}} \otimes \mathbf{t}^{\mathbf{m}} t_{0}^{-1} \mathbb{C}\left[t_{0}^{-1}\right]
\end{aligned}
$$

which are subalgebras of $\mathcal{L}$. We have

$$
\mathcal{L}=\mathcal{L}^{\geq 0} \oplus \mathcal{L}^{-}
$$

Let $\chi: \widehat{G}_{+} \rightarrow \mathbb{C}^{\times}$be the group homomorphism, i.e., a linear character of $\widehat{G}_{+}$, uniquely determined by

$$
\chi\left(\hat{\sigma}_{i}\right)=\omega_{N_{i}} \quad \text { for } 1 \leq i \leq r .
$$

More generally, for any $\mathbf{m}=\left(m_{1}, \ldots, m_{r}\right) \in \mathbb{Z}^{r}$, we define $\chi^{\mathbf{m}}$ to be the linear character of $\widehat{G}_{+}$, uniquely determined by

$$
\chi^{\mathbf{m}}\left(\hat{\sigma}_{i}\right)=\omega_{N_{i}}^{m_{i}} \quad \text { for } 1 \leq i \leq r .
$$

It can be readily seen that for $\mathbf{m}, \mathbf{n} \in \mathbb{Z}^{r}, \chi^{\mathbf{m}}=\chi^{\mathbf{n}}$ if and only if $\mathbf{m}-\mathbf{n} \in \Lambda(\mathbf{N})$. For $\mathbf{n} \in \mathbb{Z}^{r}$, using the character $\chi^{\mathbf{n}}$ we have

$$
\mathfrak{g}_{\mathbf{n}}=\left\{a \in \mathfrak{g} \mid \gamma(a)=\chi^{\mathbf{n}}(\gamma) a \quad \text { for } \gamma \in \widehat{G}_{+}\right\} .
$$

It follows that for $\mathbf{m}, \mathbf{n} \in \mathbb{Z}^{r}$ with $\mathbf{m}-\mathbf{n} \in \Lambda(\mathbf{N})$, we have $\mathfrak{g}_{\mathbf{m}}=\mathfrak{g}_{\mathbf{n}}$. For any $[\mathbf{n}] \in \mathbb{Z}^{r} / \Lambda(\mathbf{N})$ with $\mathbf{n} \in \mathbb{Z}^{r}$, we define $\mathfrak{g}_{[\mathbf{n}]}=\mathfrak{g}_{\mathbf{n}}$. Then

$$
\mathfrak{g}=\bigoplus_{[\mathbf{n}] \in \mathbb{Z}^{r} / \Lambda(\mathbf{N})} \mathfrak{g}_{[\mathbf{n}]}
$$

For $a \in \mathfrak{g}$ and $\mathbf{m} \in \mathbb{Z}^{r}$, set

$$
a_{(\mathbf{m})}=\frac{1}{N_{+}} \sum_{\gamma \in \widehat{G}_{+}} \chi^{\mathbf{m}}\left(\gamma^{-1}\right) \gamma(a),
$$


where $N_{+}=N_{1} N_{2} \cdots N_{r}=\left|\widehat{G}_{+}\right|$. For $a \in \mathfrak{g}_{\mathbf{n}}$ with $\mathbf{n} \in \mathbb{Z}^{r}$, we have $a_{(\mathbf{m})}=a$ for $\mathbf{m} \in \mathbf{n}+\Lambda(\mathbf{N})$ and $a_{(\mathbf{m})}=0$ for $\mathbf{m} \notin \mathbf{n}+\Lambda(\mathbf{N})$. Then $\mathfrak{g}_{\mathbf{m}}=\left\{a_{(\mathbf{m})} \mid a \in \mathfrak{g}\right\}$.

For $a \in \mathfrak{g},\left(m_{0}, \mathbf{m}\right) \in \mathbb{Z} \times \mathbb{Z}^{r}$, set

$$
a^{\mathcal{L}}\left(m_{0}, \mathbf{m}\right)=a_{(\mathbf{m})} \otimes t_{0}^{m_{0}} \mathbf{t}^{\mathbf{m}} \in \mathcal{L} .
$$

Furthermore, for $a \in \mathfrak{g}$, set

$$
\begin{aligned}
a^{\mathcal{L}}\left(x_{0}, \mathbf{x}\right) & =\sum_{\left(m_{0}, \mathbf{m}\right) \in \mathbb{Z} \times \mathbb{Z}^{r}} a^{\mathcal{L}}\left(m_{0}, \mathbf{m}\right) x_{0}^{-m_{0}-1} \mathbf{x}^{-\mathbf{m}} \\
& =\sum_{\left(m_{0}, \mathbf{m}\right) \in \mathbb{Z} \times \mathbb{Z}^{r}}\left(a_{(\mathbf{m})} \otimes t_{0}^{m_{0}} \mathbf{t}^{\mathbf{m}}\right) x_{0}^{-m_{0}-1} \mathbf{x}^{-\mathbf{m}} .
\end{aligned}
$$

In particular, if $a \in \mathfrak{g}_{\mathbf{k}}$ with $\mathbf{k} \in \mathbb{Z}^{r}$, we have

$$
a^{\mathcal{L}}\left(x_{0}, \mathbf{x}\right)=\sum_{m_{0} \in \mathbb{Z}, \mathbf{m} \in \mathbf{k}+\Lambda(\mathbf{N})}\left(a \otimes t_{0}^{m_{0}} \mathbf{t}^{\mathbf{m}}\right) x_{0}^{-m_{0}-1} \mathbf{x}^{-\mathbf{m}}
$$

Let $a \in \mathfrak{g}_{\mathbf{k}}, b \in \mathfrak{g}_{\mathbf{l}}$ with $\mathbf{k}, \mathbf{l} \in \mathbb{Z}^{r}$. Then

$$
\left[a^{\mathcal{L}}\left(x_{0}, \mathbf{m}\right), b^{\mathcal{L}}\left(y_{0}, \mathbf{y}\right)\right]=\mathbf{y}^{\mathbf{m}}\left([a, b]^{\mathcal{L}}\left(y_{0}, \mathbf{y}\right) x_{0}^{-1} \delta\left(\frac{y_{0}}{x_{0}}\right)+\langle a, b\rangle \mathfrak{c} \frac{\partial}{\partial y_{0}} x_{0}^{-1} \delta\left(\frac{y_{0}}{x_{0}}\right)\right)
$$

for $\mathbf{m} \in \mathbf{k}+\Lambda(\mathbf{N}) \subset \mathbb{Z}^{r}$. Note that $\left[a^{\mathcal{L}}\left(x_{0}, \mathbf{m}\right), b^{\mathcal{L}}\left(y_{0}, \mathbf{y}\right)\right]=0$ for $\mathbf{m} \notin \mathbf{k}+\Lambda(\mathbf{N})$.

An $\mathcal{L}$-module $W$ is said to be of level $\ell \in \mathbb{C}$ if $\mathfrak{c}$ acts on $W$ as scalar $\ell$ and $W$ is called a restricted module if

$$
a^{\mathcal{L}}\left(x_{0}, \mathbf{x}\right) \in \mathcal{E}(W, r) \text { for all } a \in \mathfrak{g} \text {. }
$$

We have:

Lemma 4.2. Let $W$ be any restricted $\tau$-module of level $\ell \in \mathbb{C}$. Then $\left\langle U_{W}\right\rangle$ is a restricted $\mathcal{L}$-module of level $\ell$ with $a^{\mathcal{L}}\left(y_{0}, \mathbf{y}\right)$ acting as $Y_{\mathcal{E}}\left(a^{\tau}\left(x_{0}, \mathbf{x}\right) ; y_{0}, \mathbf{y}\right)$ for $a \in \mathfrak{g}$ and with $\mathfrak{c}$ acting as scalar $\ell$. Furthermore, $U_{W}+\mathbb{C} 1_{W}$ is an $\mathcal{L}^{\geq 0}$-submodule of $\left\langle U_{W}\right\rangle$, where for $a \in \mathfrak{g}_{\mathbf{k}}, b \in \mathfrak{g}, j \in \mathbb{N}, \mathbf{m} \in \mathbf{k}+\Lambda(\mathbf{N}) \subset \mathbb{Z}^{r}$,

$$
\begin{aligned}
& \left(a \otimes t_{0}^{j} \mathbf{t}^{\mathbf{m}}\right) \cdot 1_{W}=a\left(x_{0}, \mathbf{x}\right)_{j, \mathbf{m}}^{\tau} 1_{W}=0, \\
& \left(a \otimes t_{0}^{j} \mathbf{t}^{\mathbf{m}}\right) \cdot b^{\tau}\left(x_{0}, \mathbf{x}\right)=a^{\tau}\left(x_{0}, \mathbf{x}\right)_{j, \mathbf{m}} b^{\tau}\left(x_{0}, \mathbf{x}\right)= \begin{cases}{[a, b]^{\tau}\left(x_{0}, \mathbf{x}\right)} & \text { if } j=0 \\
\langle a, b\rangle \ell 1_{W} & \text { if } j=1 \\
0 & \text { if } j \geq 2 .\end{cases}
\end{aligned}
$$


Proof. Let $a \in \mathfrak{g}_{\mathbf{k}}, b \in \mathfrak{g}$ with $\mathbf{k} \in \mathbb{Z}^{r}$ and let $\mathbf{m} \in \mathbf{k}+\Lambda(\mathbf{N}) \subset \mathbb{Z}^{r}$. Combining (4.15) with Corollary 3.7, we get

$$
\begin{aligned}
& {\left[Y_{\mathcal{E}}\left(a^{\tau}\left(t_{0}, \mathbf{t}\right) ; x_{0}, \mathbf{m}\right), Y_{\mathcal{E}}\left(b^{\tau}\left(t_{0}, \mathbf{t}\right) ; y_{0}, \mathbf{y}\right)\right] } \\
= & \mathbf{y}^{\mathbf{m}}\left(Y_{\mathcal{E}}\left([a, b]^{\tau}\left(t_{0}, \mathbf{t}\right) ; y_{0}, \mathbf{y}\right) x_{0}^{-1} \delta\left(\frac{y_{0}}{x_{0}}\right)+\langle a, b\rangle \ell \frac{\partial}{\partial y_{0}} x_{0}^{-1} \delta\left(\frac{y_{0}}{x_{0}}\right)\right) .
\end{aligned}
$$

On the other hand, notice that from (3.5), we have $Y_{\mathcal{E}}\left(a^{\tau}\left(t_{0}, \mathbf{t}\right) ; x_{0}, \mathbf{m}\right)=0$ for $\mathbf{m} \notin \mathbf{k}+\Lambda(\mathbf{N})$. Then the first assertion follows. From Corollary 3.7, the second assertion also follows.

Notice that $\widehat{L_{r+1}}(\mathfrak{g})$ is a $\mathbb{Z}$-graded Lie algebra with $\operatorname{deg}\left(a \otimes t_{0}^{k} \mathbf{t}^{\mathbf{m}}\right)=-k$ for $k \in \mathbb{Z}, \mathbf{m} \in \mathbb{Z}^{r}$ and $\operatorname{deg} \mathfrak{c}=0$, where $\mathcal{L}, \mathcal{L}^{\geq 0}$, and $\mathcal{L}^{-}$are all $\mathbb{Z}$-graded subalgebras. From Lemma 4.1 of [LTW2], we immediately have:

Lemma 4.3. Let $\ell$ be a complex number. Then there exists an $\mathcal{L}^{\geq 0}$-module structure on $\mathfrak{g} \oplus \mathbb{C}$ with $\mathfrak{c}$ acting as scalar $\ell$ and with

$$
\begin{aligned}
& \left(a \otimes t_{0}^{j} \mathbf{t}^{\mathbf{m}}\right) \cdot \mathbb{C}=0, \\
& \left(a \otimes t_{0}^{j} \mathbf{t}^{\mathbf{m}}\right) \cdot b= \begin{cases}{[a, b]} & \text { if } j=0 \\
\langle a, b\rangle \ell & \text { if } j=1 \\
0 & \text { if } j \geq 2\end{cases}
\end{aligned}
$$

for $a \in \mathfrak{g}_{\mathbf{k}}, b \in \mathfrak{g}, j \in \mathbb{N}, \mathbf{m} \in \mathbf{k}+\Lambda(\mathbf{N}) \subset \mathbb{Z}^{r}$. Furthermore, $\mathfrak{g} \oplus \mathbb{C}$ is an $\mathbb{N}$-graded $\mathcal{L}^{\geq 0}$-module with $\operatorname{deg} \mathbb{C}=0$ and $\operatorname{deg} \mathfrak{g}=1$.

For $\ell \in \mathbb{C}$, denote by $(\mathfrak{g} \oplus \mathbb{C})_{\ell}$ the $\mathcal{L}^{\geq 0}$-module obtained in Lemma 4.3. Then form an induced module

$$
V_{\mathcal{L}}(\ell, 0)=U(\mathcal{L}) \otimes_{U(\mathcal{L} \geq 0)}(\mathfrak{g} \oplus \mathbb{C})_{\ell} .
$$

In view of the $\mathrm{P}-\mathrm{B}-\mathrm{W}$ theorem, we have

$$
V_{\mathcal{L}}(\ell, 0)=U\left(\mathcal{L}^{-}\right) \otimes(\mathfrak{g} \oplus \mathbb{C})
$$

as a vector space. Consequently, $V_{\mathcal{L}}(\ell, 0)$ is an $\mathbb{N}$-graded $\mathcal{L}$-module. It follows that $V_{\mathcal{L}}(\ell, 0)$ is a restricted $\mathcal{L}$-module of level $\ell$. Set

$$
\mathbf{1}=1 \otimes 1 \in V_{\mathcal{L}}(\ell, 0) .
$$

Identify $\mathfrak{g}$ with the subspace $1 \otimes \mathfrak{g}$ through the map $a \mapsto 1 \otimes a$.

Corollary 4.4. Let $W$ be a restricted $\tau$-module of level $\ell$. Then there exists an $\mathcal{L}$-module homomorphism

$$
\phi_{W}: V_{\mathcal{L}}(\ell, 0) \rightarrow\left\langle U_{W}\right\rangle \subset \mathcal{E}\left(W, r ; N_{0}\right)
$$

such that

$$
\phi_{W}(a+\mu)=a^{\tau}\left(x_{0}, \mathbf{x}\right)+\mu 1_{W} \quad \text { for } a \in \mathfrak{g}, \mu \in \mathbb{C} .
$$


Proof. It follows from Lemma 4.2 that there exists an $\mathcal{L}^{\geq 0}$-module homomorphism $\phi_{W}^{0}$ from $(\mathfrak{g} \oplus \mathbb{C})_{\ell}$ to $\left\langle U_{W}\right\rangle$ such that

$$
\phi_{W}^{0}(a+\mu)=a^{\tau}\left(x_{0}, \mathbf{x}\right)+\mu 1_{W} \quad \text { for } a \in \mathfrak{g}, \mu \in \mathbb{C} .
$$

Then from the construction of $V_{\mathcal{L}}(\ell, 0)$, we see that $\phi_{W}^{0}$ extends uniquely to an $\mathcal{L}$-module homomorphism $\phi_{W}: V_{\mathcal{L}}(\ell, 0) \rightarrow\left\langle U_{W}\right\rangle \subset \mathcal{E}\left(W, r ; N_{0}\right)$, as desired.

We have:

Theorem 4.5. Let $\ell$ be any complex number. Then there exists an $(r+1)$-toroidal vertex algebra structure on $V_{\mathcal{L}}(\ell, 0)$, which is uniquely determined by the conditions that 1 is the vacuum vector and that

$$
Y\left(a ; x_{0}, \mathbf{x}\right)=a^{\mathcal{L}}\left(x_{0}, \mathbf{x}\right) \quad \text { for } a \in \mathfrak{g} .
$$

Proof. Since $V_{\mathcal{L}}(\ell, 0)=U(\mathcal{L})(\mathfrak{g}+\mathbb{C} 1)$, the uniqueness follows immediately. In the following, we establish the existence by applying Theorem 3.10 in [LTW2]. Temporarily, take $\sigma_{0}=1$ (with $N_{0}=1$ ) and $W=V_{\mathcal{L}}(\ell, 0)$ in (4.32). Then we have $\tau=\mathcal{L}$ and

$$
a^{\tau}\left(x_{0}, \mathbf{x}\right)=a^{\mathcal{L}}\left(x_{0}, \mathbf{x}\right) \in \mathcal{E}\left(V_{\mathcal{L}}(\ell, 0), r\right) \quad \text { for } a \in \mathfrak{g} .
$$

From Corollary 4.4, we have an $\mathcal{L}$-homomorphism $\phi_{W}$ from $V_{\mathcal{L}}(\ell, 0)$ to $\left\langle U_{W}\right\rangle$, which we alternatively denote by $\phi_{x_{0}, \mathbf{x}}$. Define

$$
\begin{aligned}
Y\left(\cdot ; x_{0}, \mathbf{x}\right): \quad & V_{\mathcal{L}}(\ell, 0) \rightarrow \mathcal{E}\left(V_{\mathcal{L}}(\ell, 0), r\right) \\
& v \mapsto \phi_{x_{0}, \mathbf{x}}(v) .
\end{aligned}
$$

For $a \in \mathfrak{g}$, we have

$$
Y\left(a ; x_{0}, \mathbf{x}\right)=\phi_{x_{0}, \mathbf{x}}(a)=a^{\mathcal{L}}\left(x_{0}, \mathbf{x}\right) .
$$

Then $\left\{Y\left(a ; x_{0}, \mathbf{x}\right) \mid a \in \mathfrak{g}\right\} \cup\left\{1_{W}\right\}$ is local. For $a \in \mathfrak{g}, v \in V_{\mathcal{L}}(\ell, 0)$, we have

$$
\begin{aligned}
& Y\left(Y\left(a ; z_{0}, \mathbf{z}\right) v ; y_{0}, \mathbf{y}\right) \\
= & \phi_{y_{0}, \mathbf{y}}\left(a^{\mathcal{L}}\left(z_{0}, \mathbf{z}\right) v\right) \\
= & Y_{\mathcal{E}}\left(a^{\mathcal{L}}\left(y_{0}, \mathbf{y}\right) ; z_{0}, \mathbf{z}\right) \phi_{y_{0}, \mathbf{y}}(v) \\
= & \operatorname{Res}_{x_{0}}\left(z_{0}^{-1} \delta\left(\frac{x_{0}-y_{0}}{z_{0}}\right) a^{\mathcal{L}}\left(x_{0}, \mathbf{z y}\right) \phi_{y_{0}, \mathbf{y}}(v)-z_{0}^{-1} \delta\left(\frac{y_{0}-x_{0}}{-z_{0}}\right) \phi_{y_{0}, \mathbf{y}}(v) a^{\mathcal{L}}\left(x_{0}, \mathbf{z y}\right)\right) \\
= & \operatorname{Res}_{x_{0}}\left(z_{0}^{-1} \delta\left(\frac{x_{0}-y_{0}}{z_{0}}\right) Y\left(a ; x_{0}, \mathbf{z y}\right) Y\left(v ; y_{0}, \mathbf{y}\right)\right. \\
& \left.\quad-z_{0}^{-1} \delta\left(\frac{y_{0}-x_{0}}{-z_{0}}\right) Y\left(v ; y_{0}, \mathbf{y}\right) Y\left(a ; x_{0}, \mathbf{z y}\right)\right) .
\end{aligned}
$$

It then follows fromTheorem 3.10 in [LTW2] that $\left(V_{\mathcal{L}}(\ell, 0), Y, \mathbf{1}\right)$ carries the structure of an $(r+1)$-toroidal vertex algebra. 
Recall that $\mathfrak{g} \oplus \mathbb{C} \subset V_{\mathcal{L}}(\ell, 0)$ and $\sigma_{0}, \ldots, \sigma_{r}$ are automorphisms of $\mathfrak{g}$.

Lemma 4.6. For each $0 \leq i \leq r$, there is an automorphism $\tilde{\sigma}_{i}$ of $V_{\mathcal{L}}(\ell, 0)$, which extends the automorphism $\sigma_{i}$ of $\mathfrak{g}$ uniquely.

Proof. Let $i \in\{0,1, \ldots, r\}$. As $V_{\mathcal{L}}(\ell, 0)$ as an $(r+1)$-toroidal vertex algebra is generated by $\mathfrak{g}$ and $\mathbf{1}$, the uniqueness is clear. It remains to establish the existence. Since the automorphism $\sigma_{i}$ of $\mathfrak{g}$ preserves the bilinear form, we can and we should view $\sigma_{i}$ as an automorphism of the Lie algebra $\widehat{L_{r+1}}(\mathfrak{g})$ by

$$
\sigma_{i}\left(a \otimes t_{0}^{m_{0}} \mathbf{t}^{\mathbf{m}}+\mu \mathfrak{c}\right)=\sigma_{i}(a) \otimes t_{0}^{m_{0}} \mathbf{t}^{\mathbf{m}}+\mu \mathfrak{c}
$$

for $a \in \mathfrak{g},\left(m_{0}, \mathbf{m}\right) \in \mathbb{Z} \times \mathbb{Z}^{r}, \mu \in \mathbb{C}$. With all $\sigma_{j}(0 \leq j \leq r)$ commuting, $\sigma_{i}$ preserves the subalgebra $\mathcal{L}$. This induces an automorphism of the universal enveloping algebra $\mathcal{U}(\mathcal{L})$, also denoted by $\sigma_{i}$. It is clear that $\sigma_{i}$ preserves the subalgebra $\mathcal{L}^{\geq 0}$.

Recall from Lemma 4.3 the $\mathcal{L}^{\geq 0}$-module structure on $\mathfrak{g} \oplus \mathbb{C}$. Let $\bar{\sigma}_{i}$ be the linear endomorphism of $\mathfrak{g} \oplus \mathbb{C}$, defined by

$$
\bar{\sigma}_{i}(a+\lambda)=\sigma_{i}(a)+\lambda \quad \text { for } a \in \mathfrak{g}, \lambda \in \mathbb{C} .
$$

From the definition of the action of $\mathcal{L}^{\geq 0}$ in Lemma 4.3, we have

$$
\bar{\sigma}_{i}(X \cdot v)=\sigma_{i}(X) \bar{\sigma}_{i}(v) \quad \text { for } X \in \mathcal{L}^{\geq 0}, v \in \mathfrak{g} \oplus \mathbb{C} .
$$

Furthermore, this holds for all $X \in U\left(\mathcal{L}^{\geq 0}\right)$. By using this property, it is straightforward to show that the linear automorphism $\sigma_{i} \otimes \bar{\sigma}_{i}$ of $U(\mathcal{L}) \otimes(\mathfrak{g} \oplus \mathbb{C})$ reduces to a linear automorphism of $V_{\mathcal{L}}(\ell, 0)$, which we denote by $\tilde{\sigma}_{i}$. We have $\tilde{\sigma}_{i}(a+\mu \mathbf{1})=\sigma_{i}(a)+\mu \mathbf{1}$ for $a \in \mathfrak{g}, \mu \in \mathbb{C}$ and $\tilde{\sigma}_{i}(X v)=\sigma_{i}(X) \tilde{\sigma}_{i}(v)$ for $X \in \mathcal{U}(\mathcal{L}), v \in V_{\mathcal{L}}(\ell, 0)$. In particular, we have

$$
\tilde{\sigma}_{i}\left(a_{m_{0}, \mathbf{m}} v\right)=\tilde{\sigma}_{i}\left(a \otimes t_{0}^{m_{0}} \mathbf{t}^{\mathbf{m}} v\right)=\sigma_{i}(a) \otimes t_{0}^{m_{0}} \mathbf{t}^{\mathbf{m}} \tilde{\sigma}(v)=\sigma_{i}(a)_{m_{0}, \mathbf{m}} \tilde{\sigma}_{i}(v)
$$

for $a \in \mathfrak{g}_{\mathbf{m}}, m_{0} \in \mathbb{Z}, \mathbf{m} \in \mathbb{Z}^{r}$. Since $V_{\mathcal{L}}(\ell, 0)$ as an $(r+1)$-toroidal vertex algebra is generated by $\mathfrak{g}$ and $\mathbf{1}$, it follows that $\tilde{\sigma}_{i}$ is an automorphism of $(r+1)$-toroidal vertex algebra $V_{\mathcal{L}}(\ell, 0)$. With $\sigma_{i}$ of order $N_{i}$, we see that $\tilde{\sigma}_{i}$ is also of order $N_{i}$.

Now, we are in a position to present the main result of this paper.

Theorem 4.7. Let $\ell \in \mathbb{C}$. For any restricted $\tau$-module $W$ of level $\ell$, there exists a $\tilde{\sigma}_{0}$-twisted $V_{\mathcal{L}}(\ell, 0)$-module structure on $W$, which is uniquely determined by

$$
Y_{W}\left(a ; x_{0}, \mathbf{x}\right)=a^{\tau}\left(x_{0}, \mathbf{x}\right) \quad \text { for } a \in \mathfrak{g}
$$

and which satisfies the condition that

$$
Y_{W}\left(\sigma_{i}(v) ; x_{0}, \mathbf{x}\right)=\lim _{x_{i} \rightarrow \omega_{i}^{-1} x_{i}} Y_{W}\left(v ; x_{0}, \mathbf{x}\right)
$$

for $v \in V_{\mathcal{L}}(\ell, 0), 1 \leq i \leq r$. On the other hand, for any $\tilde{\sigma}_{0}$-twisted $V_{\mathcal{L}}(\ell, 0)$-module $\left(W, Y_{W}\right)$ which satisfies 4 4.35), $W$ is a restricted $\tau$-module of level $\ell$ with

$$
a^{\tau}\left(x_{0}, \mathbf{x}\right)=Y_{W}\left(a ; x_{0}, \mathbf{x}\right) \quad \text { for } a \in \mathfrak{g} .
$$


Proof. From Corollary 3.6, $W$ is a faithful $\sigma$-twisted $\left\langle U_{W}\right\rangle$-module. To show that $W$ is a $\tilde{\sigma}_{0}$-twisted $V_{\mathcal{L}}(\ell, 0)$-module, we next show that there exists an $(r+1)$-toroidal vertex algebra homomorphism $\psi: V_{\mathcal{L}}(\ell, 0) \rightarrow\left\langle U_{W}\right\rangle$ such that $\psi \circ \tilde{\sigma}_{0}=\sigma \circ \psi$.

From Corollary 4.4, we have an $\mathcal{L}$-module homomorphism $\phi_{W}$ from $V_{\mathcal{L}}(\ell, 0)$ to $\left\langle U_{W}\right\rangle$. Notice that

$$
\phi_{W}(a)=a^{\tau}\left(x_{0}, \mathbf{x}\right) \quad \text { for } a \in \mathfrak{g} .
$$

Then for any $a \in \mathfrak{g}, v \in V_{\mathcal{L}}(\ell, 0)$, we have

$$
\begin{aligned}
& \phi_{W}\left(Y\left(a ; z_{0}, \mathbf{z}\right) v\right)=\phi_{W}\left(a^{\mathcal{L}}\left(z_{0}, \mathbf{z}\right) v\right) \\
& \quad=Y_{\mathcal{E}}\left(a^{\tau}\left(x_{0}, \mathbf{x}\right) ; z_{0}, \mathbf{z}\right) \phi_{W}(v)=Y_{\mathcal{E}}\left(\phi_{W}(a) ; z_{0}, \mathbf{z}\right) \phi_{W}(v) .
\end{aligned}
$$

From Lemma 2.10 in [LTW2], we see that $\phi_{W}$ is an $(r+1)$-toroidal vertex algebra homomorphism from $V_{\mathcal{L}}(\ell, 0)$ to $\left\langle U_{W}\right\rangle$.

For $a \in \mathfrak{g}_{s, \mathbf{s}}$ with $(s, \mathbf{s}) \in \mathbb{Z} \times \mathbb{Z}^{r}$, as $a^{\tau}\left(x_{0}, \mathbf{x}\right) \in \mathcal{E}\left(W, r ; N_{0}\right)_{s}$, we have

$$
\phi_{W}\left(\tilde{\sigma}_{0}(a)\right)=\omega_{N}^{s} \phi_{W}(a)=\omega_{N}^{s} a^{\tau}\left(x_{0}, \mathbf{x}\right)=\sigma\left(\phi_{W}(a)\right) .
$$

Since $V_{\mathcal{L}}(\ell, 0)$ is generated by $\mathfrak{g}$ and $\mathbf{1}$, it follows that $\phi_{W} \circ \tilde{\sigma}_{0}=\sigma \circ \phi_{W}$. Then $\phi_{W}$ is an $(r+1)$-toroidal vertex algebra homomorphism that we need. Consequently, $W$ is a $\tilde{\sigma}_{0}$-twisted $V_{\mathcal{L}}(\ell, 0)$-module with $Y_{W}\left(a ; x_{0}, \mathbf{x}\right)=a^{\tau}\left(x_{0}, \mathbf{x}\right)$ for $a \in \mathfrak{g}$. Let $a \in \mathfrak{g}_{\mathbf{m}}$ with $\mathbf{m} \in \mathbb{Z}^{r}$. For $1 \leq i \leq i$, we have

$$
\left(\sigma_{i} a\right)^{\tau}\left(x_{0}, \mathbf{x}\right)=\omega_{i}^{m_{i}} a^{\tau}\left(x_{0}, \mathbf{x}\right)=\lim _{x_{i} \rightarrow \omega_{i}^{-1} x_{i}} a^{\tau}\left(x_{0}, \mathbf{x}\right) .
$$

As $\mathfrak{g}+\mathbb{C}$ generates $V_{\mathcal{L}}(\ell, 0)$ as a toroidal vertex algebra, using the twisted iterate formula (2.14) we obtain (4.35).

Conversely, let $\left(W, Y_{W}\right)$ be a $\tilde{\sigma}_{0}$-twisted $V_{\mathcal{L}}(\ell, 0)$-module. From the construction of $(r+1)$-toroidal vertex algebra $V_{\mathcal{L}}(\ell, 0)$, we have

$$
a_{j, \mathbf{m}} b= \begin{cases}{[a, b]} & \text { if } j=0 \\ \langle a, b\rangle \ell \mathbf{1} & \text { if } j=1 \\ 0 & \text { if } j \geq 2\end{cases}
$$

for $a \in \mathfrak{g}_{\mathbf{s}}, b \in \mathfrak{g}, \mathbf{m} \in \mathbf{s}+\Lambda(\mathbf{N}) \subset \mathbb{Z}^{r}$. Then using (2.13) we obtain

$$
\begin{aligned}
& {\left[Y_{W}\left(a ; x_{0}, \mathbf{m}\right), Y_{W}\left(b ; y_{0}, \mathbf{y}\right)\right] } \\
= & \mathbf{y}^{\mathbf{m}} Y_{W}\left([a, b] ; y_{0}, \mathbf{y}\right) x_{0}^{-1} \delta\left(\frac{y_{0}}{x_{0}}\right)\left(\frac{y_{0}}{x_{0}}\right)^{\frac{s}{N}}+\mathbf{y}^{\mathbf{m}}\langle a, b\rangle \ell \frac{\partial}{\partial y_{0}}\left(x_{0}^{-1} \delta\left(\frac{y_{0}}{x_{0}}\right)\left(\frac{y_{0}}{x_{0}}\right)^{\frac{s}{N}}\right)
\end{aligned}
$$

for $\mathbf{m} \in \mathbf{s}+\Lambda(\mathbf{N})$. On the other hand, from (4.35) we have $Y_{W}\left(a ; x_{0}, \mathbf{m}\right)=0$ for $\mathbf{m} \notin \mathbf{s}+\Lambda(\mathbf{N})$. Combining these with (4.15), we conclude that $W$ is a restricted $\tau$-module of level $\ell$ with $a^{\tau}\left(x_{0}, \mathbf{x}\right)=Y_{W}\left(a ; x_{0}, \mathbf{x}\right)$ for $a \in \mathfrak{g}$. 


\section{References}

[AABGP] B. N. Allison, S. Azam, S. Berman, Y. Gao and A. Pianzola, Extended affine Lie algebras and their root systems, Memoirs Amer. Math. Soc. 126, 1997.

[ABFP] B. N. Allison, S. Berman, J. Faulkner and A. Pianzola, Multiloop realization of extended affine Lie algebras and Lie tori, Trans. Amer. Math. Soc. 361, (2009), 4807-4842.

[BBS] S. Berman, Y. Billig and J. Szmigielski, Vertex operator algebras and the representation theory of toroidal algebras, Contemporary Math. 297, Amer. Math. Soc., Providence, 2002, 1-26.

[BGK] S. Berman, Y. Gao and Y. Krylyuk, Quantum tori and the structure of elliptic quasi- simple Lie algebras, J. Funct. Anal. 135 (1996), 339-389.

[BGKN] S. Berman, Y. Gao, Y. Krylyuk and E. Neher, The alternative torus and the structure of elliptic quasi-simple Lie algebras of type A2, Trans. Amer. Math. Soc. 347 (1995), 4315-4363.

[DL] C. Dong, J. Lepowsky, Generalized Vertex Algebras and Relative Vertex Operators, Progr. Math., Vol. 112, Birkhäuser, Boston, 1993.

[FLM] I. B. Frenkel, J. Lepowsky and A. Meurman, Vertex Operator Algebras and the Monster, Pure and Applied Math., Vol. 134, Academic Press, Boston, 1988.

[FZ] I. Frenkel and Y.-C. Zhu, Vertex operator algebras associated to representations of affine and Virasoro algebras, Duke Math. J. 66 (1992), 123-168.

[IKU] T. Inami, H. Kanno, and T. Ueno, Higher-dimensional WZW Model on Käler Manifold and Toroidal Lie Algebra, Mod. Phys. Lett. A12 (1997), 2757-2764.

[IKUX] T. Inami, H. Kanno, T. Ueno, and C.-S. Xiong, Two-toroidal Lie Algebra as Current Algebra of Four-dimensional Khler WZW Model, Phys. Lett. B399 (1997), 97-104.

[K] V. G. Kac, Infinite-dimensional Lie Algebras, 3rd ed., Cambridge Univ. Press, Cambridge, 1990.

[Li1] H.-S. Li, Local systems of vertex operators, vertex superalgebras and modules, J. Pure Appl. Algebra 109 (1996), 143-195. 
[Li2] H.-S. Li, Local systems of twisted vertex operators, vertex superalgebras and twisted modules, in: Moonshine, the Monster and Related Topics, Proc. Joint Summer Research Conference, Mount Holyoke, 1994, in: C. Dong, G. Mason (Eds.), Contemp. Math. 193, Amer. Math. Soc., Providence, 1996, 203-236.

[LL] J. Lepowsky and H.-S. Li, Introduction to Vertex Operator Algebras and Their Representations, Progress in Math., Vol. 227, Birkhäuser, Boston, 2004.

[LTW1] H.-S. Li, S. Tan and Q. Wang, Twisted modules for quantum vertex algebras, J. Pure Appl. Algebra 214 (2010), 201-220.

[LTW2] H.-S. Li, S. Tan and Q. Wang, Toroidal vertex algebras and their modules, J. Algebra 365 (2012), 50-82.

[N1] E. Neher, Lie tori, C.R. Math. Acad. Sci. Soc. R. Can. 26 (2004), 84-89.

[N2] E. Neher, Extended affine Lie algebras, C.R. Math. Acad. Sci. Soc. R. Can. 26 (2004), 90-96.

[Y] Y. Yoshii, Coordinate algebras of extended affine Lie algebras of type A1, J. Algebra 234 (2000), 128-168. 